\title{
ORTHOGONAL POLYNOMIALS AND FLUCTUATIONS OF RANDOM MATRICES
}

\author{
TIMOTHY KUSALIK ${ }^{(\ddagger)}$, JAMES A. MINGO ${ }^{(*)}$, \\ AND ROLAND SPEICHER ${ }^{(*)(\dagger)}$
}

\begin{abstract}
In this paper we establish a connection between the fluctuations of Wishart random matrices, shifted Chebyshev polynomials, and planar diagrams whose linear span form a basis for the irreducible representations of the annular Temperly-Lieb algebra.
\end{abstract}

\section{INTRODUCTION}

Wishart matrices are a family of matrices studied in the statistics literature since 1928. Besides the Gaussian random matrices they constitute the most important random matrix ensemble. They can be described as follows. Let $G_{M, N}$ be a $M \times N$ matrix whose entries are independent complex Gaussian random variables with mean 0 and complex variance $1 / N$. Let $X_{N}=G_{M, N}^{*} G_{M, N} . X_{N}$ is a complex Wishart matrix (of parameter $c=M / N$ ).

The fundamental quantities of interest for random matrix ensembles are the asymptotic eigenvalue distribution and the fluctuations around this asymptotics. Whereas the main questions about eigenvalue distributions have been mostly answered decades ago, investigations around fluctuations are more recent and there is currently a lot of interest in this topic, in particular, in connection with the question of universality. In the case of Wishart matrices, the large $N$ limit of the eigenvalue distribution was found in 1967 by Marchenko and Pastur and is now named after them. The question of fluctuations was addressed for the first time by Jonsson [JON] in 1982 and, much more recently and much more detailed, by Cabanal-Duvillard [CD] in 2001.

\footnotetext{
$\ddagger$ Research supported by a USRA from the Natural Sciences and Engineering Research Council of Canada.

* Research supported by Discovery Grants and a Leadership Support Initiative Award from the Natural Sciences and Engineering Research Council of Canada.

$\dagger$ Research supported by a Premier's Research Excellence Award from the Province of Ontario.
} 
Before we say more about their results let us first describe the general picture. In the following we will, for better legibility, systematically suppress the index $N$ (or $M$ ) at our random matrices. It is to be understood that random matrices are $N \times N$-matrices and asymptotic statements refer to the limit $N \rightarrow \infty$. For many random matrices $Y$ the family of random variables $\left\{\operatorname{Tr}\left(Y^{n}\right)\right\}_{n}$ becomes asymptotically, as the size $N$ of the matrices goes to infinity, Gaussian. The two questions addressed above consist then in understanding the limit of the expectation and of the covariance of these Gaussian random variables. For the latter one would in particular like to diagonalize it. Whereas the expectation (i.e., the eigenvalue distribution) depends on the considered ensemble, the covariance (i.e., the fluctuations) seem to be much more universal. There are quite large classes of random matrices which show the same fluctuations. The most important class is the one which is represented by the Gaussian random matrices. Its fluctuations are diagonalized by Chebyshev polynomials, see Johansson [JOH]

In the case of the Wishart matrices $X$, the asymptotic Gaussianity of the traces was shown by Jonsson; the explicit form of the covariance, however, was revealed only recently by Cabanal-Duvillard [CD]. He found polynomials $\left\{\Gamma_{n}\right\}_{n}$, which were shown to be shifted Chebyshev polynomials, such that the random variables $\left\{\operatorname{Tr}\left(\Gamma_{n}(X)\right\}_{n}\right.$ are asymptotically Gaussian and independent in the large $N$ limit; that is the polynomials $\left\{\Gamma_{n}\right\}_{n}$ diagonalize asymptotically the covariance. Cabanal-Duvillard's approach relies heavily on stochastic calculus. In this paper we want to give a combinatorial proof of his results which rests on a combinatorial interpretation for the polynomials $\Gamma_{n}$. This combinatorial approach allows very canonically an extension of Cabanal-Duvillard's results to a family of independent Wishart matrices, yielding our main result.

Theorem Let $\left\{\Gamma_{n}\right\}_{n}$ be the shifted Chebyshev polynomials of the first kind as considered by Cabanal-Duvillard and let $\left\{\Pi_{n}\right\}_{n}$ be the orthogonal polynomials of the Marchenko-Pastur distribution (which are shifted Chebyshev polynomials of the second kind). Let $X_{1}, \ldots, X_{p}$ be independent Wishart matrices and consider in addition to $\operatorname{Tr}\left(\Gamma_{n}\left(X_{i}\right)\right)$ also, for $k \geq 2$, the collection of random variables $\operatorname{Tr}\left(\Pi_{m_{1}}\left(X_{i_{1}}\right) \cdots \Pi_{m_{k}}\left(X_{i_{k}}\right)\right)$, where the Wishart matrices which appear must be cyclically alternating, i.e., $i_{1} \neq i_{2} \neq i_{3} \neq \cdots \neq i_{k} \neq i_{1}$. These latter traces depend only on the equivalence class of $\left(i_{1}, \ldots, i_{k}\right)$ and $\left(m_{1}, \ldots m_{k}\right)$ under cyclic permutation. Assuming that we have chosen one representative from each equivalence class, the random variables

$$
\left\{\operatorname{Tr}\left(\Gamma_{n}\left(X_{i}\right)\right)\right\} \cup\left\{\operatorname{Tr}\left(\Pi_{m_{1}}\left(X_{i_{1}}\right) \cdots \Pi_{m_{k}}\left(X_{i_{k}}\right)\right)\right\}
$$


are asymptotically independent and Gaussian.

Let us remark that, in contrast to one-matrix models, multi-matrix models are very poorly understood and the problem of universality is mostly open for them at the moment. Understanding the fluctuations of the simplest representatives of multi-matrix models is essential for progress in this direction. Whereas our results about the multimatrix Wishart case are new, the corresponding results for the multimatrix Gaussian case were derived by Cabanal-Duvillard in [CD]. By a small modification of our approach we can also give a combinatorial re-interpretation and proof in this case (see the remarks in Section 11).

The main motivation for our investigations comes from our belief that the theory of free probability provides the right tools and concepts for attacking questions on fluctuations of random matrices - in particular, for multi-matrix models. Even though "freeness" will not appear explicitly in this paper, our methods and results are very much related to our investigations around "second order freeness" in [MS]. The present paper can, in particular, be seen as a complementary treatment of some of the questions treated in [MS].

In the rest of this Introduction we want to give some idea of what is involved in the proof of our theorem; in particular, we would like to outline the relation between special planar diagrams and the question of diagonalizing the covariance of Wishart matrices.

Let us start with our re-interpretation of Cabanal-Duvillard's results for the case of one Wishart matrix. Our starting point is the paper of Mingo-Nica [MN], where a genus expansion in terms of permutations was provided for the cumulants of the random variables $\operatorname{Tr}\left(X^{n}\right)$. Since cumulants of different orders have different leading contributions in $N$, this has as a direct consequence the asymptotic Gaussianity of these traces. The main problem left is to understand and diagonalize the covariance. Also in [MN], it was shown that the covariance of the random variables $\left\{\operatorname{Tr}\left(X^{n}\right)\right\}_{n}$ has asymptotically a very nice combinatorial interpretation, namely it is given by counting a class of planar diagrams which were called non-crossing annular permutations. More precisely, if we denote by $\kappa_{2}(A, B)$ the covariance of two random variables $A$ and $B$ and if $c$ is the asymptotic ratio of $M$ and $N$ for our Wishart matrices, we have

$$
\lim _{N \rightarrow \infty} \kappa_{2}\left(\operatorname{Tr}\left(X^{m}\right), \operatorname{Tr}\left(X^{n}\right)\right)=\sum_{\pi \in S_{N C}(m, n)} c^{\# \pi},
$$

where $S_{N C}(m, n)$ denotes the set of non-crossing $(m, n)$-annular permutations, i.e., permutations on $m+n$ points which connect $m$ points on one circle with $n$ points on another circle in a planar or non-crossing 
way. In the above formula we are summing over all non-crossing $(m, n)$ annular permutations and each block of such a permutation contributes a multiplicative factor $c$. (For $c=1$, which corresponds to Wishart matrices with $M=N$, the above formula just counts the number of elements in $S_{N C}(m, n)$.)

In [MN] the cycles of the permutation were shown as blocks in the annulus. See the figure below.

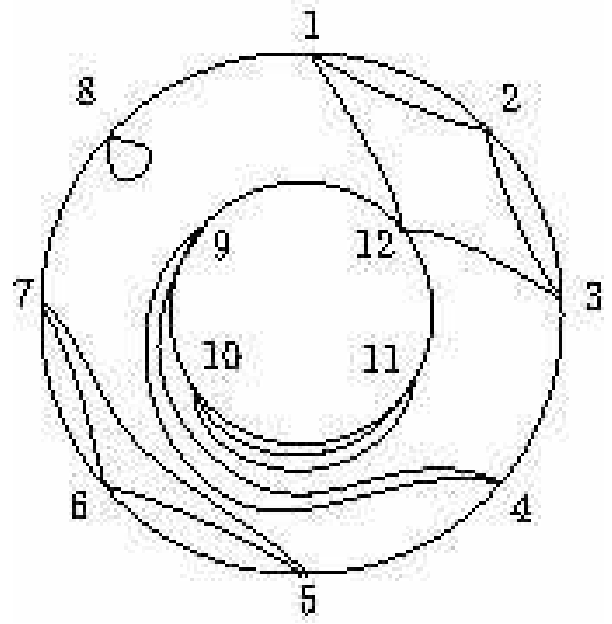

Figure 1. A non-crossing annular permutation.

In our context it seems more appropriate to redraw the diagram with the circles side by side as shown.

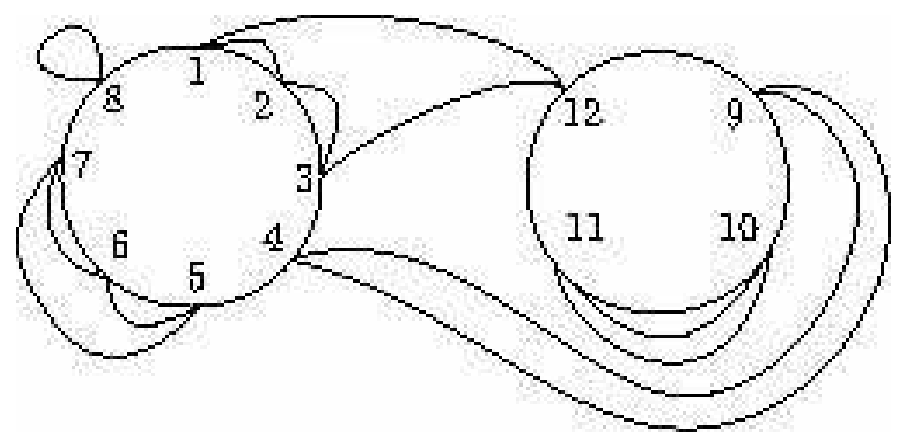

Figure 2. The permutation of figure 1 unfolded.

The limit of $\kappa_{2}\left(\operatorname{Tr}\left(X^{m}\right), \operatorname{Tr}\left(X^{n}\right)\right)$ is, of course, not diagonal in $m$ and $n$ because points on each circle are grouped into blocks (some of which do not even connect to the other circle), and this grouping on both sides has no correlation; so there is no constraint that $m$ has to be equal to $n$. However, a quantity which clearly must be the same for both circles is the number of through-blocks, i.e., blocks which connect both circles. Thus in order to diagonalize the covariance we should go over from the number of points on a circle to the number of through-blocks leaving this circle. 
A nice way to achieve this is to cut our diagrams in two parts - one part for each circle as shown.
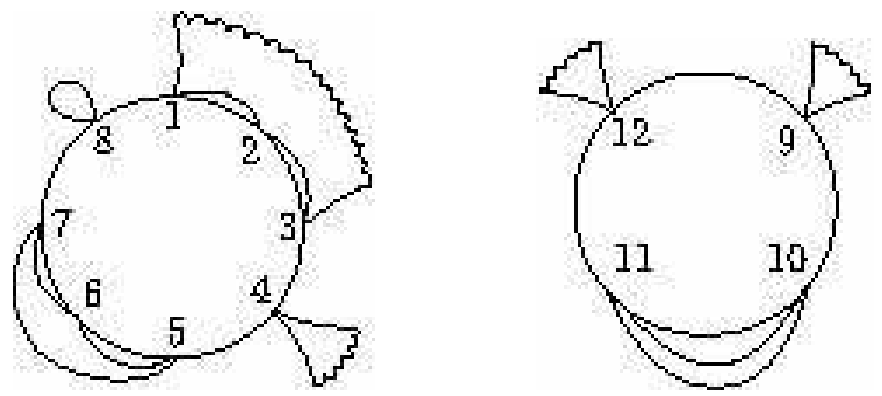

Figure 3. The permutation of figure 2 cut in two.

These diagrams which we call 'non-crossing half-permutations' are the principal objects of study of this paper.

In this pictorial description $\operatorname{Tr}\left(X^{m}\right)$ corresponds to the sum over non-crossing half-permutations on one circle with $m$ points and $\operatorname{Tr}\left(X^{n}\right)$ corresponds to a sum over non-crossing half-permutations on another circle with $n$ points. The limit of $\kappa_{2}\left(\operatorname{Tr}\left(X^{m}\right), \operatorname{Tr}\left(X^{n}\right)\right)$ corresponds to pairing the half-permutations for $\operatorname{Tr}\left(X^{m}\right)$ with the half-permutations for $\operatorname{Tr}\left(X^{n}\right)$. The pairing between two half-permutations is given by glueing them together in all possible planar ways. We will call what is left in a half-permutation of a through-block after cutting an 'open' block - as opposed to 'closed' blocks which live totally on one circle and are thus not affected by the cutting. A pairing of two half-permutations consists of glueing together their open blocks in all possible planar ways. This clearly means that both half-permutations must have the same number of open blocks for a non-trivial pairing, and thus our covariance should become diagonal if we go over from the number of points on a circle to the number of open blocks. From this point of view the polynomials $\Gamma_{k}(x)$ found by Cabanal-Duvillard should describe $k$ open blocks. If we write $x^{n}$ as a linear combination of the $\Gamma_{k}$ 's as in

$$
x^{n}=\sum_{k=0}^{n} q_{n, k} \Gamma_{k}(x)
$$

then the above correspondence

$$
\begin{aligned}
\operatorname{Tr}\left(X^{n}\right) & \hat{=} n \text { points } \\
\operatorname{Tr}\left(\Gamma_{k}(X)\right) & \hat{=} \quad k \text { open blocks }
\end{aligned}
$$

suggests that the coefficients $q_{n, k}$ are given by summing over all halfpermutations with $k$ open blocks, each such permutation contributing a factor $c$ for each closed block. (The dependence on $c$ reflects the fact that in our original formula every block contributed with a factor $c-$ 
now every closed block gives a factor $c$ right away, whereas an open block has to be paired with another open block to produce a factor $c$. In the case $c=1, q_{n, k}$ just counts the number of half-permutations on $n$ points which have $k$ open blocks.) We will show that the coefficients $q_{n, k}$ appearing in the relations for the shifted Chebyshev polynomials $\Gamma_{k}$ have indeed this combinatorial meaning. This will be achieved, in Section 8, by showing that both quantities satisfy the same recurrence relations. Let us also point out that the case $k=0$ is special, because constant terms in the polynomials play no role for the covariance, but have to be fixed by other normalizations. On the combinatorial level this is reflected by the fact that we only look on non-crossing annular permutations which connect the two circles, thus we always have at least $k \geq 1$ through-blocks (or open blocks, after cutting). Since however, our recursions rely also on $k=0$, we have to make some separate considerations for $k=0$ at various places. In particular, we want to point out that the 'right' definition in our setting for a non-crossing half-permutation on $n$ points with zero open block is not just a noncrossing permutation on $n$ points. See Section 8 for more details on this.

In order to illustrate the above statements let us here present the pictorial meaning of the equation

$$
x^{2}=\Gamma_{2}+(2+2 c) \Gamma_{1}+q_{2,0} \Gamma_{0}
$$

for the shifted Chebyshev polynomials $\Gamma_{k}$.

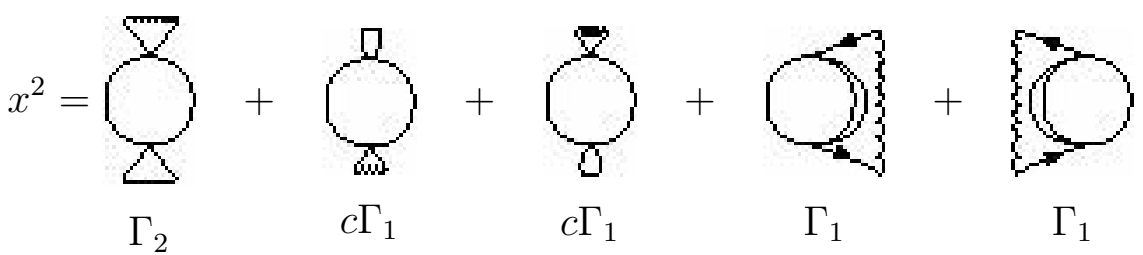

Figure 4. The five non-crossing half-permutations on [2].

The diagrams corresponding to $\Gamma_{0}$ have been omitted.

Our combinatorial interpretation of the diagonalizing polynomials for one Wishart matrix opens the way to a similar treatment for a tuple of independent Wishart matrices. Covariances of traces of products of such matrices are asymptotically described essentially in the same way as before in terms of non-crossing annular permutations, however, in addition we have to colour the points on the two circles (one colour for each Wishart matrix) and require that the contributing permutations have to connect only points of the same colour (i.e., each cycle of the permutation must be mono-chromatic). Again the diagonalization of 
the covariance is achieved by going over from the number of points on a circle to the number of open blocks. Thus, on first view, one might expect that traces of alternating products in the $\Gamma_{k}$ give rise to a diagonal covariance. However, this is not the case. One has to realize that through-blocks of one colour break the symmetry of the circle for through-blocks of another colour, thus in a sense for each group of through-blocks of the same colour the circle is cut open to a line and instead of circular half-permutations we have to consider linear halfpermutations. Thus instead of $\Gamma_{k}$ we have to look for polynomials $\Pi_{k}$ which satisfy

$$
x^{n}=\sum_{k=0}^{n} p_{n, k} \Pi_{k}(x),
$$

where $p_{n, k}$ is now the sum over all linear half-permutations with $k$ open blocks, each closed block weighted by a factor $c$. It turns out that these polynomials are the orthogonal polynomials for the Marchenko-Pastur distribution (which are shifted Chebyshev polynomials of the second kind). Again we prove this, in Section 7, by showing the equality of the corresponding recurrence relations. The proof that the covariance is diagonalized by traces in alternating products in these polynomials $\Pi_{k}$ is given in Section 9 .

As an illustrative example for these statements consider

$$
x^{2} y=\left\{\Pi_{2}(x)+(1+2 c) \Pi_{1}(x)+\left(c+c^{2}\right) \Pi_{0}(x)\right\}\left\{\Pi_{1}(y)+c \Pi_{0}(y)\right\}
$$

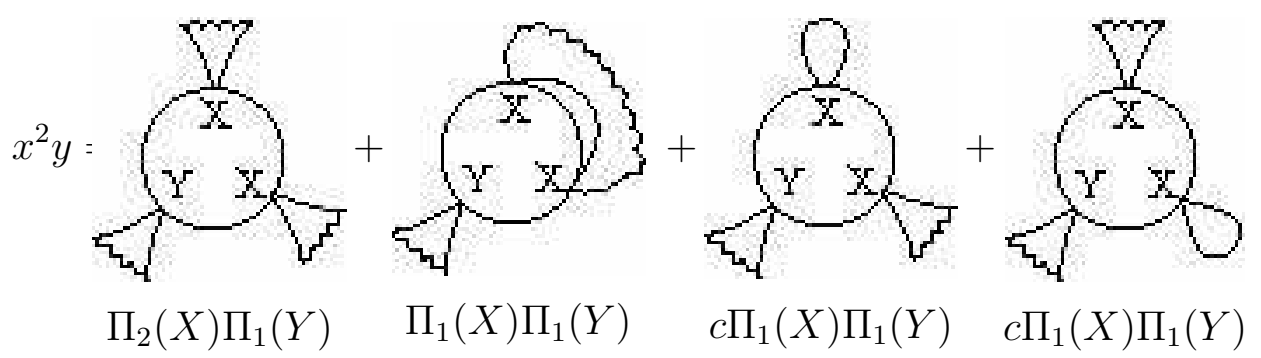

Figure 5. The four non-crossing circular half-permutations on $\{X, X, Y\}$. The diagrams corresponding to $\Pi_{0}$ have been omitted.

Note that the above argument of symmetry breaking is strictly valid only if each group of the same colour has at least one through-block. However, a priori one also has to consider diagrams containing monochromatic groups without any through-block. It turns out that, by the centeredness of the $\Pi_{k}$, the net contribution of such diagrams cancels out. To make this argument rigourous constitutes an essential part of the proof of Theorem 1 (see in particular Lemma 37) 
Finally, we would like to point out that our circular half-permutations are, after a small redrawing, the diagrams used by V. F. R. Jones [J], $\S 5$ to create a basis for the irreducible representations of the annular Temperly-Lieb algebras. In Jones's convention the left picture in Fig. 3 above would be first inverted in the centre of the circle and then the blocks would be expanded into 'fat graphs'.
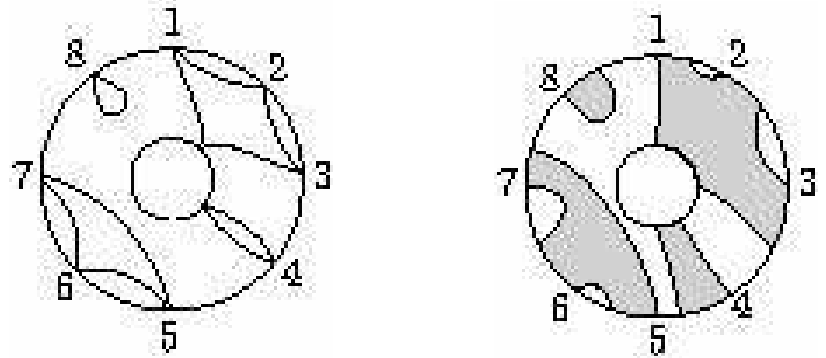

Figure 6. The left diagram of figure 3 inverted and then drawn as a fat permutation.

In this paper we will not have to say more about this relation with the Temperly-Lieb algebras, but we are taking this as a serious hint that there exists a deeper relation between free probability, random matrices, and subfactors. We hope to explore this relation further in forthcoming investigations.

However, as a nice application of our developed machinery to operator algebraic questions we show, in Section 10, a connection between Wick products and half-permutations that gives a combinatorial formula for the product of two Wick products which has a very simple diagrammatic interpretation.

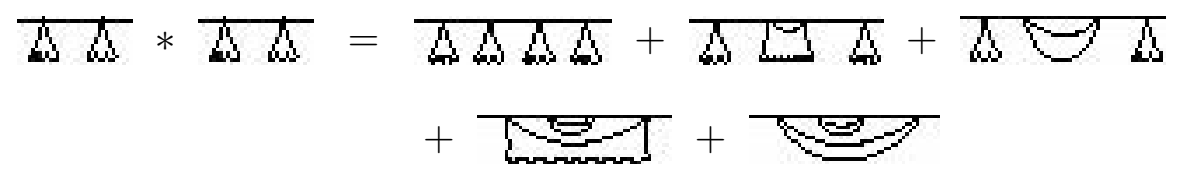

Figure 7. The convolution of two non-crossing linear half permutations.

\section{Notation}

Let $(\Omega, \Sigma, \mathrm{P})$ be a probability space. We will only consider random variables with moments of all orders. Let $G_{M, N}: \Omega \rightarrow M_{M, N}(\mathbb{C})$ be a random matrix with entries $\left(g_{i, j}\right)$ such that each $g_{i, j}$ is a complex Gaussian random variable with mean 0 and complex variance $\mathcal{E}\left(\left|g_{i, j}\right|^{2}\right)=1 / N$. Let $X_{N}=G_{M, N}^{*} G_{M, N}$, we shall call $X_{N}$ a complex Wishart distributed random matrix or more succinctly a Wishart 


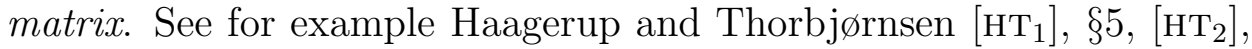
$\S 6$ or Hiai and Petz [HP], Ch. 4.

For any random variables $X$ and $Y$, let the covariance be given by $\kappa_{2}(\mathrm{X}, \mathrm{Y})=\mathcal{E}(\mathrm{XY})-\mathcal{E}(\mathrm{X}) \mathcal{E}(\mathrm{Y})$. For an $N \times N$ matrix $X$ we let $\operatorname{Tr}(X)$ be the un-normalized trace.

In this paper we are interested in the large $N$ limit of the eigenvalue distribution of $X_{N}$ and their fluctuations. In order for this limit to exist we must have that $M / N$ converges as $M$ and $N$ tend to infinity. More precisely we shall suppose that we have sequences $\left\{M_{k}\right\}_{k}$ and $\left\{N_{k}\right\}_{k}$ such that $\lim _{k} M_{k}=\lim _{k} N_{k}=\infty$ and $\lim _{k} M_{k} / N_{k}=c$ with $0<c<\infty$. In order to have a second order distribution we require in addition the existence of a second order limit $c^{\prime}=\lim _{k} M_{k}-c N_{k}$. We shall assume that the numbers $c$ and $c^{\prime}$ are fixed throughout the paper.

This limiting distribution is called the Marchenko-Pastur distribution,

$$
\mu_{c}=(1-c) \delta_{0}+\frac{\sqrt{(b-x)(x-a)}}{2 \pi x} d x
$$

where $a=(\sqrt{c}-1)^{2}, b=(\sqrt{c}+1)^{2}$, and $\delta_{0}$ is an atom at 0 which disappears if $c \geq 1$. The absolutely continuous part $\sqrt{(b-x)(x-a)} /(2 \pi x)$ has support equal to the interval $[a, b]$.

\section{Shifted Chebyshev Polynomials of the First Kind}

We shall make use of two families $\left\{\Gamma_{k}(x)\right\}_{k}$ and $\left\{\Pi_{k}(x)\right\}_{k}$ of shifted Chebyshev polynomials c.f. [CD]. Let $\left\{T_{k}(x)\right\}_{k}$ be the Chebyshev polynomials of the first kind i.e. $T_{k}(\cos \theta)=\cos (k \theta)$. Rescale these polynomials by letting $C_{0}(x)=1$ and for $n \geq 1, C_{k}(x)=2 T_{k}(x / 2)$. Then $\left\{C_{k}(x)\right\}_{k}$ are monic and orthogonal for the dilated arc-sine law $\frac{d x}{\pi \sqrt{4-x^{2}}}$ on $[-2,2]$.

We have

$$
\begin{aligned}
C_{0}(x) & =1 \\
C_{1}(x) & =x \\
C_{2}(x) & =x^{2}-2 \\
C_{3}(x) & =x^{3}-3 x \text { and } \\
x C_{n}(x) & =C_{n+1}(x)+C_{n-1}(x) \text { for } n>1
\end{aligned}
$$

Now let us shift the sequence to produce our first sequence $\left\{\widetilde{\Gamma}_{n}(x)\right\}_{n}$. Let

$$
u=\frac{x-(1+c)}{\sqrt{c}} \quad \text { then } \quad \frac{d u}{\sqrt{4-u^{2}}}=\frac{d x}{\sqrt{(b-x)(x-a)}}
$$


where $a=(\sqrt{c}-1)^{2}$ and $b=(\sqrt{c}+1)^{2}$.

Now let

$$
\widetilde{\Gamma}_{n}(x)=\sqrt{c^{n}} C_{n}\left(\frac{x-(1+c)}{\sqrt{c}}\right)
$$

Then

$$
\begin{aligned}
& \widetilde{\Gamma}_{0}(x)=1 \\
& \widetilde{\Gamma}_{1}(x)=x-(1+c) \\
& \widetilde{\Gamma}_{2}(x)=x^{2}-2(1+c) x+1+c^{2} \\
& \widetilde{\Gamma}_{3}(x)=x^{3}-3(1+c) x^{2}+3\left(1+c+c^{2}\right) x-\left(1+c^{3}\right) \\
& x \widetilde{\Gamma}_{n}(x)=\widetilde{\Gamma}_{n+1}(x)+(1+c) \widetilde{\Gamma}_{n}(x)+c \widetilde{\Gamma}_{n-1}(x) \text { for } n=0,2,3,4, \ldots
\end{aligned}
$$

and $\left\{\widetilde{\Gamma}_{n}(x)\right\}_{n}$ is a sequence of monic pairwise orthogonal polynomials for the shifted arc-sine law $\frac{d x}{\pi \sqrt{(b-x)(x-a)}}$ on $[a, b]$.

We shall write $\widetilde{\Gamma}_{n}(x)=\sum_{k=0}^{n} g_{n, k}^{\prime} x^{k}$. The recurrence formula for the $\widetilde{\Gamma}_{n}$ 's means that for $n=0,2,3,4, \ldots$ we have

$$
g_{n, k-1}^{\prime}=g_{n+1, k}^{\prime}+(1+c) g_{n, k}^{\prime}+c g_{n-1, k}^{\prime}
$$

and for $n=1$

$$
g_{1, k-1}^{\prime}=g_{2, k}^{\prime}+(1+c) g_{1, k}^{\prime}+2 c g_{0, k}
$$

where we have used the convention that $g_{n, k}^{\prime}=0$ if either $n<0$ or $k<0$, or $k>n$. Let $\widetilde{\Gamma}$ denote the upper-triangular matrix

$$
\widetilde{\Gamma}=\left(\begin{array}{ccccc}
g_{0,0}^{\prime} & & & & \\
g_{1,0}^{\prime} & g_{1,1}^{\prime} & & & \\
g_{2,0}^{\prime} & g_{2,1}^{\prime} & g_{2,2}^{\prime} & & \\
g_{3,0}^{\prime} & g_{3,1}^{\prime} & g_{3,2}^{\prime} & g_{3,3}^{\prime} & \\
& \ddots & \ddots & \ddots & \ddots
\end{array}\right)
$$

Let $S$ be the unilateral shift and $C=c S+c I+S S^{*}+S^{*}$

$$
S=\left(\begin{array}{cccc}
0 & 0 & & \\
1 & 0 & 0 & \\
& 1 & 0 & \ddots \\
& & \ddots & \ddots
\end{array}\right), C=\left(\begin{array}{cccc}
c & 1 & & \\
c & 1+c & 1 & \\
& c & 1+c & \ddots \\
& & \ddots & \ddots
\end{array}\right)
$$

Equations (11) and (2) imply that

$$
\widetilde{\Gamma} S^{*}=C \widetilde{\Gamma}+(I+c S)\left(I-S S^{*}\right) \widetilde{\Gamma}
$$


After multiplying on the left and the right by $\widetilde{\Gamma}^{-1}$ we have

$$
\widetilde{\Gamma}^{-1} C=S^{*} \widetilde{\Gamma}^{-1}-\widetilde{\Gamma}^{-1}(I+c S)\left(I-S S^{*}\right)
$$

If we write

$$
\widetilde{\Gamma}^{-1}=\left(\begin{array}{ccccc}
g_{0,0} & & & & \\
g_{1,0} & g_{1,1} & & & \\
g_{2,0} & g_{2,1} & g_{2,2} & & \\
g_{3,0} & g_{3,1} & g_{3,2} & g_{3,3} & \\
& \ddots & \ddots & \ddots & \ddots
\end{array}\right)
$$

then equation (4) implies that for $k>0$

$$
g_{n+1, k}=g_{n, k-1}+(1+c) g_{n, k}+c g_{n, k+1}
$$

and for $k=0$

$$
g_{n+1,0}=c g_{n, 1}+(1+c) g_{n, 0}+c g_{n, 1}
$$

By direct calculation we obtain that the first five rows of $\widetilde{\Gamma}^{-1}$ are

$$
\left(\begin{array}{ccccc}
1 & 0 & 0 & 0 & 0 \\
1+c & 1 & 0 & 0 & 0 \\
1+4 c+c^{2} & 2+2 c & 1 & 0 & 0 \\
1+9 c+9 c^{2}+c^{3} & 3+9 c+3 c^{2} & 3+3 c & 1 & 0 \\
1+16 c+36 c^{2}+16 c^{3}+c^{4} & 4+24 c+24 c^{2}+4 c^{3} & 6+16 c+6 c^{2} & 4+4 c & 1
\end{array}\right)
$$

Let $d_{0}=-1, d_{1}=1$ and $d_{n}=(-1)^{n}(c-1)$ for $n>1$. Let

$$
\Gamma_{n}(x)=\widetilde{\Gamma}_{n}(x)+d_{n}
$$

then $\int_{\mathbb{R}} \Gamma_{n}(x) d \mu_{c}(x)=0$ for $n=0,1,2$. By equation 4 above,

$$
\int_{\mathbb{R}} \widetilde{\Gamma}_{n}(x) d \mu_{c}(x)=(-1)^{n}(1-c)
$$

for $n>1$ and thus $\int_{\mathbb{R}} \Gamma_{n}(x) d \mu_{c}(x)=0$ for all $n$.

Let us write $\Gamma_{n}(x)=\sum_{k=0}^{n} q_{n, k}^{\prime} x^{k}$ and $x^{n}=\sum_{k=0}^{n} q_{n, k} \Gamma_{k}(x)$. Let $\Gamma$ be the matrix

$$
\Gamma=\left(\begin{array}{ccccc}
q_{0,0}^{\prime} & & & & \\
q_{1,0}^{\prime} & q_{1,1}^{\prime} & & & \\
q_{2,0}^{\prime} & q_{2,1}^{\prime} & q_{2,2}^{\prime} & & \\
q_{3,0}^{\prime} & q_{3,1}^{\prime} & q_{3,2}^{\prime} & q_{3,3}^{\prime} & \\
& \ddots & \ddots & \ddots & \ddots
\end{array}\right)
$$


and thus

$$
\Gamma^{-1}=\left(\begin{array}{ccccc}
q_{0,0} & & & & \\
q_{1,0} & q_{1,1} & & & \\
q_{2,0} & q_{2,1} & q_{2,2} & & \\
q_{3,0} & q_{3,1} & q_{3,2} & q_{3,3} & \\
& \ddots & \ddots & \ddots & \ddots
\end{array}\right)
$$

Let $D$ be the matrix

$$
D=\left(\begin{array}{c|cc}
d_{0} & 0 & \cdots \\
d_{1} & 0 & \cdots \\
d_{2} & 0 & \cdots \\
d_{3} & 0 & \cdots \\
\vdots & \vdots &
\end{array}\right)
$$

Then $\Gamma=\widetilde{\Gamma}+D$ and so $\Gamma^{-1}=\widetilde{\Gamma}^{-1}-\widetilde{\Gamma}^{-1} D$, since $D \Gamma=D$ which holds because $q_{0,0}^{\prime}=1$. Thus $q_{n, k}=g_{n, k}$ for $k>0$.

The first five rows of $\Gamma^{-1}$ are

$$
\left(\begin{array}{ccccc}
1 & 0 & 0 & 0 & 0 \\
c & 1 & 0 & 0 & 0 \\
c+c^{2} & 2+2 c & 1 & 0 & 0 \\
c+3 c^{2}+c^{3} & 3+9 c+3 c^{2} & 3+3 c & 1 & 0 \\
c+6 c^{2}+6 c^{3}+c^{4} & 4+24 c+24 c^{2}+4 c^{3} & 6+16 c+6 c^{2} & 4+4 c & 1
\end{array}\right)
$$

We shall see in section 8 that these equations have a very simple combinatorial interpretation.

Recall that the Cauchy transform of the arc-sine law is

$$
G(z)=\int_{-2}^{2} \frac{1}{z-t} \frac{d t}{\pi \sqrt{4-t^{2}}}=\frac{1}{\sqrt{z^{2}-4}}
$$

Using the transformation $u=(z-(1+c)) / \sqrt{c}$ we see that the Cauchy transform of the shifted arc-sine law is

$$
F(z)=\int_{a}^{b} \frac{1}{z-t} \frac{d t}{\pi \sqrt{(b-t)(t-a)}}=G(u) / \sqrt{c}
$$

Thus the moment generating function of the shifted arc-sine law is

$$
\sum_{n \geq 0} g_{n, 0} z^{n}=z^{-1} F\left(z^{-1}\right)=\frac{1}{\sqrt{(1-a z)(1-b z)}}
$$

Now for each $k>0$ let

$$
G_{k}(z)=\sum_{n \geq k} g_{n, k} z^{n}
$$


Then $G_{0}(z)=\frac{1}{\sqrt{(1-a z)(1-b z)}}$. From equation (5) we see that for $k \geq 1$

$$
c G_{k+1}(z)=\left(z^{-1}-(1+c)\right) G_{k}(z)-G_{k-1}(z)
$$

and

$$
2 c G_{1}(z)=\left(z^{-1}-(1+c)\right) G_{0}(z)-z^{-1}
$$

Let

$$
P_{0}(z)=\frac{1-(c-1) z-\sqrt{(1-a z)(1-b z)}}{2 z}
$$

be the moment generating function of the Marchenko-Pastur distribution. Then we can write equation (8) as

$$
G_{1}(z)=\left(\frac{P_{0}(z)-1}{c}\right) G_{0}(z)
$$

Moreover $P_{0}(z)$ satisfies the functional equation $\left(z^{-1}-(1+c)\right)\left(P_{0}(z)-\right.$ $1)=\left(P_{0}(z)-1\right)^{2}+c$. Now we can apply equation (7) to conclude by induction that

$$
G_{n}(z)=\left(\frac{P_{0}(z)-1}{c}\right)^{n} G_{0}(z)
$$

There is an interesting diagrammatic interpretation of equation (9) in section 6, c.f. Remark 30.

\section{Shifted Chebyshev Polynomials of the Second Kind}

We next recall the construction of the orthogonal polynomials for the Marchenko-Pastur distribution $\mu_{c}$. Let $\left\{U_{n}(x)\right\}_{n}$ be the Chebyshev polynomials of the second kind, i.e. $U_{n}(\cos \theta)=\sin ((n+1) \theta) / \sin \theta$. Let $S_{n}(x)=U_{n}(x / 2)$. Then

$$
\begin{array}{rlrl}
S_{0}(x) & =1 & S_{3}(x) & =x^{3}-2 x \\
S_{1}(x) & =x & x S_{n}(x) & =S_{n+1}(x)+S_{n-1}(x) \text { for } n>1 \\
S_{2}(x) & =x^{2}-1 & &
\end{array}
$$

The $S_{n}$ 's are monic and orthogonal for the semicircle law $\sqrt{4-x^{2}} /(2 \pi)$. We let

$$
\Pi_{n}(x)=\sqrt{c^{n}} S_{n}\left(\frac{x-(1+c)}{\sqrt{c}}\right)+\sqrt{c^{n-1}} S_{n-1}\left(\frac{x-(1+c)}{\sqrt{c}}\right)
$$

Then

$$
\Pi_{0}(x)=1 \quad \Pi_{2}(x)=x^{2}-(1+2 c) x+c^{2}
$$




$$
\Pi_{1}(x)=x-c \quad \Pi_{3}(x)=x^{3}-(2+3 c) x^{2}+\left(1+2 c+3 c^{2}\right) x-c^{3}
$$

and for $n>1$

$$
x \Pi_{n}(x)=\Pi_{n+1}(x)+(1+c) \Pi_{n}(x)+c \Pi_{n-1}(x)
$$

The $\Pi_{n}$ 's are shifted Chebyshev polynomial of the second kind. Indeed by letting

$$
u=\frac{x-(1+c)}{\sqrt{c}} \quad \text { then } \quad \frac{c \sqrt{4-u^{2}} d u}{2 \pi}=\frac{\sqrt{(b-x)(x-a)} d x}{2 \pi}
$$

where $a=(\sqrt{c}-1)^{2}$ and $b=(\sqrt{c}+1)^{2}$, we obtain, from equation (10), that $\int_{\mathbb{R}} x \Pi_{n}(x) d \mu_{c}(x)=0$ for $n>1$. In addition we can see by direct calculation that $\int_{\mathbb{R}} \Pi_{1}(x) d \mu_{c}(x)=\int_{\mathbb{R}} \Pi_{2}(x) d \mu_{c}(x)=0$. Thus by the recurrence relation we have that $\int_{\mathbb{R}} \Pi_{n}(x) d \mu_{c}(x)=0$ for all $n>0$. Finally by induction on $m+n$ we have again from the recurrence relation for $n>m$ that $\int_{\mathbb{R}} x^{m} \Pi_{n}(x) d \mu_{c}(x)=\int_{\mathbb{R}} x \Pi_{m+n-1}(x) d \mu_{c}(x)=0$. This shows that the $\Pi_{n}$ 's are pairwise orthogonal. From the recurrence relation we have that

$$
\int_{\mathbb{R}} \Pi_{n}(x)^{2} d \mu_{c}(x)=\int_{\mathbb{R}} x \Pi_{n}(x) \Pi_{n-1}(x) d \mu_{c}(x)
$$

and from equation (10) we obtain

$$
\int_{\mathbb{R}} x \Pi_{n}(x) \Pi_{n-1}(x) d \mu_{c}(x)=c^{n} \int_{\mathbb{R}} S_{n-1}(u)^{2} \frac{\sqrt{4-u^{2}}}{2 \pi} d u=c^{n}
$$

Thus $\int_{\mathbb{R}} \Pi_{n}(x)^{2} d \mu_{c}(x)=c^{n}$.

Let us write $\Pi_{n}(x)=\sum_{k=0}^{n} p_{n, k}^{\prime} x^{k}$ and $x^{n}=\sum_{k=0}^{n} p_{n, k} \Pi_{k}(x)$. Let $\Pi$ be the matrix

$$
\Pi=\left(\begin{array}{ccccc}
p_{0,0}^{\prime} & & & & \\
p_{1,0}^{\prime} & p_{1,1}^{\prime} & & & \\
p_{2,0}^{\prime} & p_{2,1}^{\prime} & p_{2,2}^{\prime} & & \\
p_{3,0}^{\prime} & p_{3,1}^{\prime} & p_{3,2}^{\prime} & p_{3,3}^{\prime} & \\
& \ddots & \ddots & \ddots & \ddots
\end{array}\right)
$$

and thus

$$
\Pi^{-1}=\left(\begin{array}{cccccc}
p_{0,0} & & & & \\
p_{1,0} & p_{1,1} & & & \\
p_{2,0} & p_{2,1} & q_{2,2} & & \\
p_{3,0} & p_{3,1} & p_{3,2} & q_{3,3} & \\
& \ddots & \ddots & \ddots & \ddots
\end{array}\right)
$$


Let $C$ and $S$ be as in equation (3) above. Then we have from the recurrence relation for the $\Pi$ 's that $C \Pi=\Pi S^{*}$ and thus $\Pi^{-1} C=$ $S^{*} \Pi^{-1}$. From this we obtain the recurrence relation for the $p_{n, k}$ 's:

$$
p_{n+1, k}=p_{n, k-1}+(1+c) p_{n, k}+c p_{n, k+1} \text {, when } k>0
$$

$$
\text { and } p_{n+1,0}=c p_{n, 0}+c p_{n, 1} \text {, when } k=0 \text {. }
$$

In section [7] we present a simple combinatorial interpretation for this recurrence relation. Here are the first five rows of $\Pi^{-1}$.

$$
\left(\begin{array}{ccccc}
1 & 0 & 0 & 0 & 0 \\
c & 1 & 0 & 0 & 0 \\
c+c^{2} & 1+2 c & 1 & 0 & 0 \\
c+3 c^{2}+c^{3} & 1+5 c+3 c^{2} & 2+3 c & 1 & 0 \\
c+6 c^{2}+6 c^{3}+c^{4} & 1+9 c+14 c^{2}+4 c^{3} & 3+11 c+6 c^{2} & 3+4 c & 1
\end{array}\right)
$$

We can also obtain immediately the generating functions for the sequences $\left\{p_{n, k}\right\}_{n}$ as in Haagerup-Thorbjørnsen [HT2], $\S 6$. Let $P_{k}(z)=$ $\sum_{n \geq 0} p_{n, k} z^{n}$. Then $P_{0}(z)=\frac{1-(c-1) z-\sqrt{(1-a z)(1-b z)}}{2 z}$ is the moment generating function of the Marchenko-Pastur distribution. From equation (11) we get that for $k \geq 1$

$$
c P_{k+1}(z)=\left(z^{-1}-(1+c)\right) P_{k}(z)-P_{k-1}(z)
$$

and from equation (12) we get that

$$
\left(\frac{P_{0}(z)-1}{z}\right)=c P_{0}(z)+c P_{1}(z)
$$

From this equation and the functional equation for $P_{0}$ we get that

$$
P_{1}(z)=\left(\frac{P_{0}(z)-1}{c}\right) P_{0}(z)
$$

Then by induction we get, as noted in [ $\left.\mathrm{HT}_{1}\right]$, Lemma 6.3, from (13) that for all $k$,

$$
P_{k}(z)=\left(\frac{P_{0}(z)-1}{c}\right)^{k} P_{0}(z)
$$

In Remark 19 we give a simple and elegant diagrammatic interpretation of this formula.

Finally let us note that the relation $2 T_{n}(x)=U_{n}(x)-U_{n-2}(x)$ between the Chebyshev polynomials of the first and second kind implies that

$$
\widetilde{\Gamma}_{n}(x)+\widetilde{\Gamma}_{n-1}(x)=\Pi_{n}(x)-c \Pi_{n-2}(x)
$$

for $n \geq 2$ and thus (since $d_{n}+d_{n-1}=0$ for $n \geq 3$ )

$$
\Gamma_{n}(x)+\Gamma_{n-1}(x)=\Pi_{n}(x)-c \Pi_{n-2}(x) .
$$




\section{Wishart Matrices}

Let $(\Omega, \Sigma, \mathrm{P})$ be a probability space and $G: \Omega \rightarrow M_{M, N}(\mathbb{C})$ be a random matrix with entries $\left\{g_{i, j}\right\}$. Suppose that $\left\{g_{i, j}\right\}$ are independent complex Gaussian random variables with $\mathcal{E}\left(g_{i, j}\right)=0$ and $\mathcal{E}\left(\left|g_{i, j}\right|^{2}\right)=$ $1 / N$ for all $1 \leq i \leq M$ and $1 \leq j \leq N$. Then $X=G^{*} G$ is a particular case of a complex Wishart matrix. To simplify the terminology we shall henceforth say that $X$ is a Wishart matrix if $X=G^{*} G$ and $G$ is as above for some $M$ and $N$.

We are interested in the behaviour of the eigenvalue distribution of $X_{N}$ as $M$ and $N$ tend to infinity. Thus we assume that we have sequences $\left\{M_{k}\right\}_{k}$ and $\left\{N_{k}\right\}_{k}$ of positive integers such that $c:=\lim _{k} M_{k} / N_{k}$ exists and $0<c<\infty$. In order to get a second order limiting distribution we shall further assume that the limit $c^{\prime}=\lim _{k \rightarrow \infty} M_{k}-c N_{k}$ exists. Whenever asymptotics are discussed in this paper we shall always assume that $M$ and $N$ are chosen from sequences $\left\{M_{k}\right\}_{k}$ and $\left\{N_{k}\right\}_{k}$ satisfying the two limiting behaviours above. When we take a limit as $k$ tends to infinity we shall denote this as $\lim _{N}$. For further details and references see $\left[\mathrm{HT}_{1}\right],\left[\mathrm{HT}_{2}\right]$, and [MN].

It was shown in Cabanal-Duvillard [CD] that the family of random variables $\left\{\operatorname{Tr}\left(\Gamma_{n}\left(X_{N}\right)\right)\right\}_{n>0}$ is asymptotically Gaussian and independent as $N$ tends to infinity, where the $\Gamma_{k}$ 's are the shifted Chebyshev polynomials of the first kind constructed in section 3. We wish to extend this to a collection of independent Wishart matrices. So suppose that for each $M$ and $N$ we have $G_{1}, G_{2}, \ldots, G_{p}$ each as above but in addition such that the entries of all the $G_{i}$ 's are independent. Thus for each $M$ and $N$ we have Wishart matrices $X_{1}^{(N)}, \ldots, X_{p}^{(N)}$ where $X_{i}^{(N)}=G_{i}^{*} G_{i}$. We now wish to construct for each $k$-tuple of positive integers $\vec{m}=\left(m_{1}, \ldots, m_{k}\right)$ and each sequence of indices $\vec{i}=\left(i_{1}, \ldots, i_{k}\right)$ with $1 \leq i_{j} \leq p$ and $i_{j} \neq i_{j+1}$ a random variable $S_{\vec{m}, \vec{i}}$ :

$$
S_{\vec{m}, \vec{i}}^{(N)}=\operatorname{Tr}\left(\Pi_{m_{1}}\left(X_{i_{1}}^{(N)}\right) \Pi_{m_{2}}\left(X_{i_{2}}^{(N)}\right) \cdots \Pi_{m_{k}}\left(X_{i_{k}}^{(N)}\right)\right)
$$

where the $\Pi_{k}$ 's are the shifted Chebyshev polynomials of the second kind constructed in section 4. $S_{\vec{m}, \vec{i}}^{(N)}$ only depends on the equivalence class of $(\vec{m}, \vec{\imath})$ under cyclic permutation, so in Theorem 1 below we shall assume that we have chosen one representative from each equivalence class. Let $|(\vec{m}, \vec{\imath})|$ be the number of cyclic equivalence classes, i.e. the number of $1 \leq l \leq k$ such that for $1 \leq r \leq k$ we have $m_{r}=m_{k+l+r}$ and $i_{r}=i_{k+l+r}$, where the indices are taken modulo $k$. 
Theorem 1. Let $\left\{\Gamma_{n}\right\}_{n}$ be the shifted Chebyshev polynomials of the first kind as considered by Cabanal-Duvillard and let $\left\{\Pi_{n}\right\}_{n}$ be the orthogonal polynomials of the Marchenko-Pastur distribution (which are shifted Chebyshev polynomials of the second kind). Let $X_{1}, \ldots, X_{p}$ be independent Wishart matrices and consider in addition to $\operatorname{Tr}\left(\Gamma_{n}\left(X_{i}\right)\right)$ also, for $k \geq 2$, the collection of random variables $\operatorname{Tr}\left(\Pi_{m_{1}}\left(X_{i_{1}}\right) \cdots \Pi_{m_{k}}\left(X_{i_{k}}\right)\right)$, where the Wishart matrices which appear must be cyclically alternating, i.e., $i_{1} \neq i_{2} \neq i_{3} \neq \cdots \neq i_{k} \neq i_{1}$. These latter traces depend only on the equivalence class of $\left(i_{1}, \ldots, i_{k}\right)$ and $\left(m_{1}, \ldots m_{k}\right)$ under cyclic permutation. Assuming that we have chosen one representative from each equivalence class, the random variables

$$
\left\{\operatorname{Tr}\left(\Gamma_{n}\left(X_{i}\right)\right)\right\} \cup\left\{\operatorname{Tr}\left(\Pi_{m_{1}}\left(X_{i_{1}}\right) \cdots \Pi_{m_{k}}\left(X_{i_{k}}\right)\right)\right\}
$$

are asymptotically independent and Gaussian.

Moreover the limiting means of $\operatorname{Tr}\left(\Gamma_{n}\left(X_{N}\right)\right)$ and $S_{\vec{m}, \vec{\imath}}$ are given by

$$
\begin{aligned}
\left.\lim _{N} \mathcal{E}\left(\operatorname{Tr}\left(\Gamma_{n}\left(X_{N, i}\right)\right)\right)\right) & =(-1)^{n} c^{\prime} \text { and } \\
\lim _{N} \mathcal{E}\left(S_{\vec{m}, \vec{\imath}}\right) & =0
\end{aligned}
$$

Finally the asymptotic variances of $S_{\vec{m}, \vec{i}}$ and $\operatorname{Tr}\left(\Gamma_{n}\left(X_{N}\right)\right)$ are given by

$$
\begin{gathered}
\lim _{N} \kappa_{2}\left(\operatorname{Tr}\left(\Gamma_{n}\left(X_{N, i}\right), \operatorname{Tr}\left(\Gamma_{n}\left(X_{N, i}\right)\right)\right)=n c^{n}\right. \text { and } \\
\lim _{N} \kappa_{2}\left(\operatorname{Tr}\left(S_{\vec{m}, \vec{i}}\right), \operatorname{Tr}\left(S_{\vec{m}, \vec{i}}\right)\right)=\lim _{N} \mathcal{E}\left(\left|\operatorname{Tr}\left(S_{\vec{m}, \vec{i}}\right)\right|^{2}\right)=|(\vec{m}, \vec{\imath})| c^{m_{1}+\cdots+m_{k}}
\end{gathered}
$$

Remark 2. In proving Theorem 10 we shall show along the way that

$$
\left.\lim _{N} \operatorname{Tr}\left(\Pi_{n}\left(X_{N, i}\right)\right)\right)= \begin{cases}0 & n \text { is even } \\ c^{\prime} c^{k} & n=2 k+1\end{cases}
$$

In Mingo and Nica [MN], $§ 9$ it was shown that the traces of words in $\left\{X_{i}^{(N)}\right\}_{i}$ are asymptotically Gaussian, in fact that all the cumulants of order higher than 2 are asymptotically 0 , thus the random variables $\left\{\operatorname{Tr}\left(\Gamma_{m}\left(X_{i}^{(N)}\right)\right)\right\}_{m, i}$ and $\left\{S_{\vec{m}, \vec{i}}^{(N)}\right\}_{\vec{m}, \vec{i}}$ are asymptotically Gaussian.

We shall prove Theorem 1 by showing that asymptotically $\left\{S_{\vec{m}, \vec{i}}^{(N)}\right\}_{\vec{m}, \vec{i}}$ $\cup\left\{\operatorname{Tr}\left(\Gamma_{m}\left(X_{j}^{(N)}\right)\right)\right\}_{m, i}$ are independent, i.e. that they diagonalize the covariance $\kappa_{2}$. In [MN], $\S 7$ it was shown that that these covariances can be expressed in terms of planar diagrams called non-crossing annular permutations. In the next two sections we will show how to relate these diagrams to the polynomials $\left\{\Gamma_{n}\right\}_{n}$ and $\left\{\Pi_{n}\right\}_{n}$. 


\section{Non-Crossing Circular Half-Permutations}

We introduce the notion of a non-crossing circular half-permutation. A non-crossing circular half-permutation on $[\mathrm{m}]$ is a non-crossing permutation $\pi$ of $[m]$ together with a selection of blocks of $\pi$ that satisfy a condition described below.

We shall begin by recalling the definition of non-crossing permutations. Let $[m]=\{1,2,3, \ldots, m\}$. Let $\pi$ be a partition of $[m]$. We say $\pi$ has a crossing if there are $r<s<t<u$ with $r$ and $t$ belonging to one block of $\pi$ and $s$ and $u$ belonging to another. We say $\pi$ is non-crossing if it has no crossings.

Another useful picture is to consider $\pi$ as a permutation of $[m]$. Each block of $\pi$ is arranged into increasing order and these form the cycles of a permutation of $[m]$. The permutations so obtained are characterized by the equality $\#(\pi)+\#\left(\gamma_{m} \pi^{-1}\right)=m+1$, where $\gamma_{m}$ is the permutation with one cycle $(1,2,3, \ldots, m)$ and $\#(\sigma)$ is the number of cycles in the permutation $\sigma$; see Biane $[\mathrm{B}]$. As there is a bijection between permutations of $[m]$ satisfying this geodesic condition and partitions satisfying the non-crossing condition we shall denote them both by $\pi$. When it is necessary to emphasize the distinction we shall denote the non-crossing partitions by $N C(m)$ and the non-crossing permutations by $S_{N C}(m)$.

Perhaps the simplest description however is in terms of planar diagrams. Given a partition $\pi$ we place the numbers $1,2,3, \ldots, m$ around a circle in clockwise order, and in the interior of the circle connect the points in the same block. If this can be drawn so that the blocks do not cross then the partition is non-crossing.

Given a non-crossing partition $\pi$ there is another partition called the Kreweras complement which we shall denote by $\pi^{c}$. The complement can be described several ways. First, let us regard $\pi$ as a partition of $[m]$. Let us consider another set $[\bar{m}]=\{\overline{1}, \overline{2}, \ldots, \bar{m}\}$ and arrange the union $[\bar{m}] \cup[m]$ in the order $\overline{1}<1<\overline{2}<2<\cdots<\bar{m}<m$. Then $\pi^{c}$ is the largest partition of $[\bar{m}]$ such that $\pi^{c} \cup \pi$ is a non-crossing partition of $[\bar{m}] \cup[m]$.

Alternatively we can regard $\pi$ as a permutation of $[m]$ and then $\pi^{c}=\gamma_{m} \pi^{-1}$, see Biane [B].

Definition 3. A non-crossing circular half-permutation of $[m]$ is a tuple $\left(\pi, \bar{B}, B_{1}, B_{2}, \ldots, B_{k}\right)$ where $\pi$ is a non-crossing permutation of $[\mathrm{m}]$, $k \geq 1, B_{1}, \ldots B_{k}$ are blocks of $\pi$, and $\bar{B}$ is a block of $\pi^{c}$ such that $B_{i} \cap \bar{B} \neq \varnothing$ for $i=1, \ldots, k$. The blocks $B_{1}, \ldots, B_{k}$ are called the open blocks of $\pi$ and the remaining blocks of $\pi$ are called the closed blocks. Frequently and when no confusion can occur we shall simply 
denote $\left(\pi, \bar{B}, B_{1}, \ldots, B_{k}\right)$ by $\pi$. The set of non-crossing circular halfpermutations with $k$ open blocks will be denoted $N C C(m)_{k}$.

Lemma 4. Suppose that $\pi \in N C(m), B$ is a block of $\pi, \bar{B}$ is a block of $\pi^{c}$ such that $B \cap \bar{B} \neq \varnothing$. Then $|B \cap \bar{B}|=1$.

Proof. Suppose $i, j \in B \cap \bar{B}$ with $i<j$. For this proof we shall use the picture of $\pi^{c}$ as a partition on $[\bar{m}]$ such that $\pi^{c} \cup \pi$ is a non-crossing partition of $[\bar{m}] \cup[m]$. Then $\bar{i}<i<\bar{j}<j$ and $i, j \in B$ and $\bar{i}, \bar{j} \in \bar{B}$ which gives a crossing of $\pi^{c} \cup \pi$. Hence $|B \cap \bar{B}|=1$.

Definition 5. Let $\pi \in N C(m), B \in \pi$, and $\bar{B} \in \pi^{c}$ with $B \cap \bar{B}=\{i\}$. Then $i$ is called the initial point of $B$ relative to $\bar{B}$. We call $\pi^{-1}(i)$ the final point of $B$ relative to $\bar{B}$. If $B$ is a singleton then its initial and final points will coincide.

We can now present another picture of non-crossing circular halfpermutations that explains the terminology we have introduced above.

In [MN] it was shown that for a sequence of Wishart matrices $\left\{X_{N}\right\}$ the correlation of the moments

$$
\mathcal{E}\left(\operatorname{Tr}\left(X_{N}^{m}\right) \operatorname{Tr}\left(X_{N}^{n}\right)\right)-\mathcal{E}\left(\operatorname{Tr}\left(X_{N}^{m}\right)\right) \mathcal{E}\left(\operatorname{Tr}\left(X_{N}^{n}\right)\right)
$$

converged as $N$ tends to infinity, to $\sum_{\pi \in S_{N C}(m, n)} c^{\#(\pi)} . S_{N C}(m, n)$ is the collection of non-crossing annular permutations on the $(m, n)$-annulus and $\#(\pi)$ is the number of cycles of $\pi$. These were the subject of [MN]; but we shall recall the pertinent facts here.

The notion of a non-crossing annular partition extends to the annulus the idea of a non-crossing partition on a disc. Given two integers $m$ and $n$ and two concentric circles we have on the outer one the points $1,2, \ldots, m$ in clockwise order and on the inner circle we have the points $m+1, \ldots, m+n$ in counter-clockwise order. We call this the $(m, n)$-annulus. We want to study partitions of $[m+n]$ such that when drawn on the $(m, n)$-annulus there is at least one block connecting the two circles and the blocks do not cross. Being non-crossing on the $(m, n)$-annulus is weaker than being non-crossing on the $m+n$-disc.

Given a non-crossing partition of the disc we can always put the points of each block in standard order and obtain a permutation satisfying Biane's condition. In the case of the annulus we have to distinguish between permutations whose orbits as a set are the same but which visit the points in different orders.

Informally a permutation $\pi$ in $S_{m+n}$ is non-crossing on the $(m, n)$ annulus if we can connect the points in cycles in the order visited by $\pi$ 
in such a way that the blocks do not cross or self-intersect and enclose their interior in the clockwise orientation..

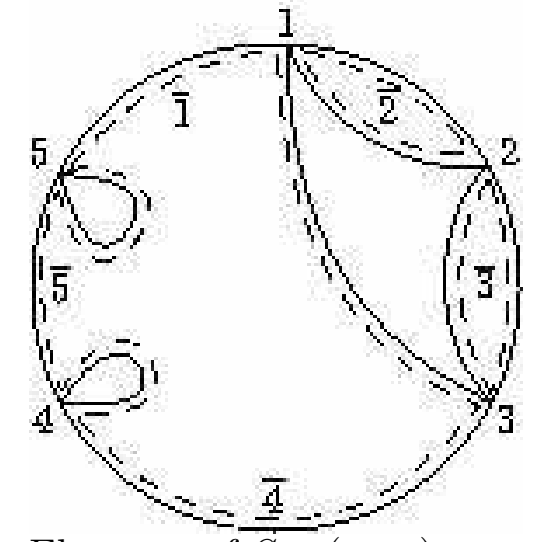

Figure 8. The non-crossing partition $\pi=(1,2,3)(4)(5)$ and its complement $\pi^{c}=(1,4,5)(2)(3)$.

Elements of $\dot{S}_{N C}(m, n)$ are permutations of $[m+n]$ which satisfy an annular geodesic condition similar to the one found by Biane. Let $\gamma_{m, n}$ be the permutation of $[m+n]$ with two cycles

$$
\gamma_{m, n}=(1,2,3, \ldots, m)(m+1, \ldots, m+n)
$$

A permutation $\pi$ is non-crossing annular if it connects the two circles and

$$
\#(\pi)+\#\left(\gamma_{m, n} \pi^{-1}\right)=m+n
$$

There are a variety of equivalent ways of expressing this condition; see [MN], $\S 3,4,5,6$ and [MS], $\S 2$.

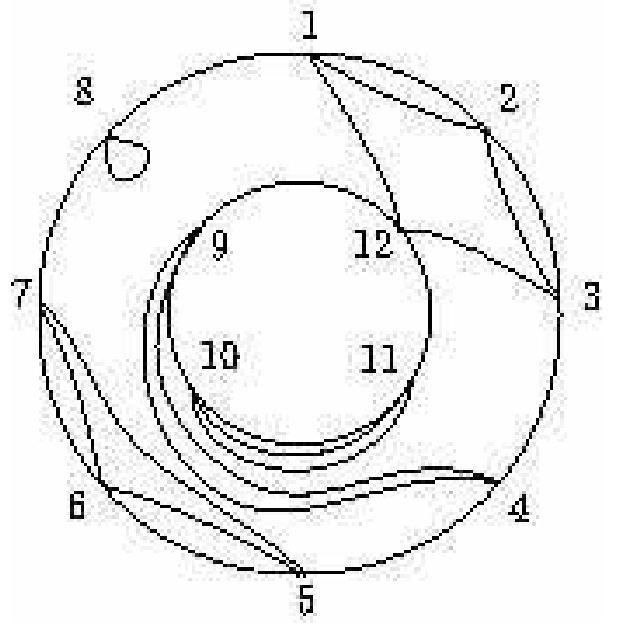

Figure 9. $\pi=(1,2,3,12)(4,9)$ $(5,6,7)(8)(10,11)$ is a non-crossing annular $(8,4)$-permutation and its complements $\pi^{c}=(1,9,5,8)$

$(2)(3)(4,10,12)(6)(7)$.

We now wish to describe what happens if we cut one of these annular diagrams into two pieces which we shall call half-permutations. Informally we mean we separate the two circles; blocks that lie completely on one circle are called closed blocks and blocks that connect 
the two circles are cut in half and are called open blocks. Let us consider the example $\pi=(1,2,3,12)(4,9)(5,6,7)(8)(10,11)$ which is a non-crossing permutation of the $(8,4)$-annulus. There are two throughblocks i.e. blocks that connect the two circles: $(1,2,3,12)$ and $(4,9)$. So if we consider the half on the outside circle we have the half-permutation $\{(1,2,3)(4)(5,6,7)(8)\}$. The open blocks are $(1,2,3)$ and $(4)$ and the closed blocks are $(5,6,7)$ and $(8)$.
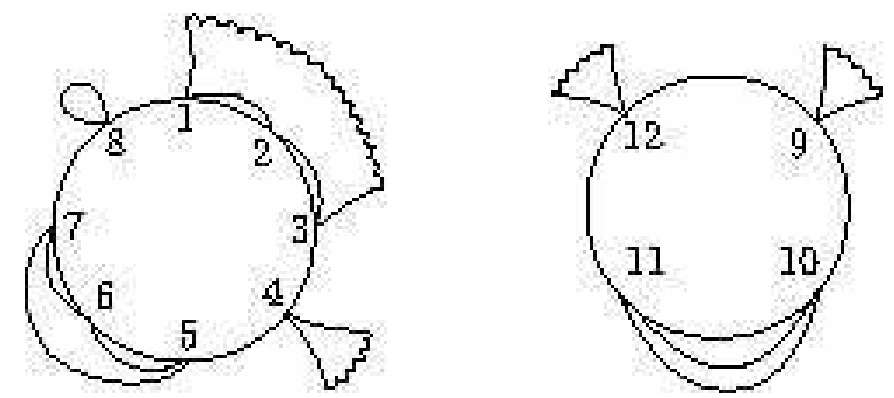

Figure 10. The two non-crossing circular half-permutations arising from $\pi=(1,2,3,12)(4,9)(5,6,7)(8)(10,11)$.

We now give a formal definition.

Notation 6. Let $S_{N C}(m, n)$ be the set of non-crossing annular permutations. For $\pi \in S_{N C}(m, n)$ we call $\gamma_{m, n} \pi^{-1}$ the annular complement of $\pi$ and denote it also by $\pi^{c} . \pi^{c}$ is also non-crossing by equation (15). For $\pi \in S_{N C}(m, n)$ and $B$ a cycle of $\pi$ we say that $B$ is through-block if $B \cap[m] \neq \varnothing$ and $B \cap[m+1, m+n] \neq \varnothing$

Notation 7. Let $\pi$ be a permutation of $[n]$ and let $A \subset[n]$. Let $\pi_{\left.\right|_{A}}$ denote the permutation induced by $\pi$ on $A$, i.e. for $i \in A, \pi_{\left.\right|_{A}}(i)=j$ if $\pi^{k}(i)=j \in A$ and $\pi^{l}(i) \notin A$ for $1 \leq l<k$.

Lemma 8. Let $\pi \in S_{N C}(m, n)$ and $\pi_{1}=\pi_{\left.\right|_{[m]}}$. Let $\bar{B}_{1}, \bar{B}_{2}, \ldots, \bar{B}_{k}$ be the through-blocks of $\pi^{c}$. Let $\bar{B}=\left(\bar{B}_{1} \cup \cdots \cup \bar{B}_{k}\right) \cap[m]$, then $\bar{B}$ is a cycle of $\pi_{1}^{c}$.

Proof. We must show that $\bar{B}$ is invariant under $\pi_{1}^{c}$ and that $\pi_{1}^{c}$ acts transitively on $\bar{B}$. Let $i \in \bar{B}$. First suppose $\pi^{c}(i) \in[m]$, then $\pi^{-1}(i) \in$ $[m]$ and so $\pi_{1}^{-1}(i)=\pi^{-1}(i)$; thus $\pi_{1}^{c}(i)=\gamma_{m} \pi_{1}^{-1}(i)=\pi^{c}(i) \in \bar{B}$.

Suppose $\pi^{c}(i) \in[m+1, m+n]$, then $\pi^{-1}(i) \in[m+1, m+n]$. Let $k$ be such that $\pi^{-k}(i) \in[m]$ but $\pi^{-l}(i) \in[m+1, m+n]$ for $1 \leq l<k$. Let $j=\pi^{-k+1}(i)$. Then $\gamma_{m, n} \pi^{-1}(j)=\gamma_{m} \pi_{1}^{-1}(i)$. So $\pi_{1}^{c}(i)$ is in a throughblock of $\pi^{c}$ and thus $\pi_{1}^{c}(i) \in \bar{B}$. Hence $\bar{B}$ is invariant under $\pi_{1}^{c}$.

If $\bar{B}_{i}$ is a through-block of $\pi^{c}$ and $i \in \bar{B}_{i}$ is such that $\left(\pi^{c}\right)^{-1}(i) \in$ $[m+1, m+n]$ then we shall call $i$ the initial point of $\bar{B}_{i}$; conversely if 
$\pi^{c}(i) \in[m+1, m+n]$ then we shall call $i$ the final point of $\bar{B}_{i}$. We have just seen in the first paragraph of the proof that if $i \in \bar{B}_{i}$ is not the final point then $\pi_{1}^{c}$ moves through the points of $\bar{B}_{i} \cap[m]$ until it comes to the final point of $\bar{B}_{i}$. In the second paragraph we saw that $\pi_{1}^{c}$ takes the final point of one block of $\pi^{c}$ to the initial point of another block.

Each of the blocks $\bar{B}_{i}$, being a through-block, has a non-empty intersection with $[m+n]$. By property $(\mathrm{AC}-3)$ of $[\mathrm{MN}]$, Def. 3.5, applied to $\pi^{c}$ we see that once $\gamma_{m}$ visits a block $\bar{B}_{i}$ it doesn't visit it again until all the other blocks $\bar{B}_{j}(j \neq i)$ have been visited. So let us order the blocks $\bar{B}_{i}$ so that $\gamma_{m}$ visits the blocks in the order $\bar{B}_{1}, \bar{B}_{2}, \ldots, \bar{B}_{k}$. If $i \in \bar{B}_{r} \cap[m]$ and $i$ is a final point of $\bar{B}_{i}$ then $i$ is an initial point of a block $C$ of $\pi$ and so $\pi_{1}^{-1}(i)$ is the final point of $C$; thus $\gamma_{m} \pi_{1}^{-1}(i)$ is the initial point of $\bar{B}_{r+1}$.

Definition 9. Let $\pi$ be a non-crossing annular $(m, n)$-permutation. We shall construct a pair $\pi_{1}$ and $\pi_{2}$ of non-crossing circular halfpermutations from $\pi$.

Let $\pi_{1}=\pi_{\left.\right|_{[m]}}$. By [MN], Def. 3.5, (AC-1) $\pi_{1}$ is non-crossing.

Suppose the through-blocks of $\pi^{c}$ are $\bar{B}_{1}, \bar{B}_{2}, \ldots, \bar{B}_{r}$. Let $\bar{B}=$ $\left(\bar{B}_{1} \cup \cdots \cup \bar{B}_{r}\right) \cap[m]$. Let $B_{1}, B_{2}, \ldots, B_{s}$ be the through-blocks of $\pi$. By Lemma 8, $\bar{B}$ is a cycle of $\pi_{1}^{c}$ and the blocks $B_{i} \cap[m]$ all meet $\bar{B}$. Thus $\left(\pi_{1}, B_{1} \cap[m], B_{2} \cap[m], \ldots, B_{s} \cap[m], \bar{B}\right)$ is a non-crossing circular half-permutation.

Similarly let $\pi_{2}=\pi_{\left.\right|_{[m+1, m+n]}}$. Then $\pi_{2}$ is non-crossing on $[m+1, m+$ $n$. Let $\bar{B}^{\prime}=\left(\bar{B}_{1} \cup \cdots \cup \bar{B}_{r}\right) \cap[m+1, m+n]$. Then $\left(\pi_{2}, B_{1} \cap[m+1, m+\right.$ $\left.n], B_{2} \cap[m+1, m+n], \ldots, B_{s} \cap[m+1, m+n], \bar{B}^{\prime}\right)$ is a non-crossing circular half-permutation on $[m+1, m+n] . \quad \pi_{1}$ and $\pi_{2}$ are the two half-permutations obtained from $\pi$.

Definition 10. Let $\left(\sigma, B_{1}, B_{2}, \ldots, B_{k}, \bar{B}\right)$ be a non-crossing circular half-permutation of $[m]$. We shall say that the cycles $B_{1}, B_{2}, \ldots, B_{k}$ are in cyclic order if for each $i$ and each $x \in B_{i}$ and $r$ such that $\gamma_{m}^{r}(x) \in \cup_{l \neq i} B_{l}$ and $\gamma_{m}^{s}(x) \notin \cup_{l \neq i} B_{l}$ for $1 \leq s<r, \gamma_{m}^{r}(x) \in B_{i+1}$ where we interpret $B_{k+1}$ as $B_{1}$.

Lemma 11. Let $\left(\sigma, B_{1}, B_{2}, \ldots, B_{k}, \bar{B}\right)$ be a non-crossing circular halfpermutation of $[m]$. There is an ordering of the cycles $B_{1}, B_{2}, \ldots, B_{k}$ such that they are in cyclic order.

Proof. We showed in Lemma 4 that for each $i,\left|B_{i} \cap \bar{B}\right|=1$. Let us label these points $x_{1}, x_{2}, \ldots, x_{k}$, i.e. $\left\{x_{i}\right\}=\left|B_{i} \cap \bar{B}\right|$. Moreover suppose that the cycles $B_{1}, B_{2}, \ldots, B_{k}$ are ordered so that $\gamma_{m}$ visits the $x_{i}$ 's in 
the order $x_{1}, x_{2}, \ldots, x_{k}$. We shall show that this puts the cycles into cyclic order.

Choose $i$ and $x \in B_{i}$ and let $r$ be such that $\gamma_{m}^{r}(x) \in \cup_{l \neq i} B_{l}$ and $\gamma_{m}^{s}(x) \notin \cup_{l \neq i} B_{l}$ for $1 \leq s<r$. Since the points $\bar{x}_{1}, x_{1}, \bar{x}_{2}, x_{2}, \ldots, \bar{x}_{k}, x_{k}$, of $[\bar{m}] \cup[m]$, are in cyclic order we can only have $x_{i} \leq x<x_{i+1}$, otherwise there would be a crossing between $B_{i}$ and $\bar{B}$. Hence $\gamma_{m}^{r}(x)=$ $x_{i+1} \in B_{i+1}$.

Notation 12. Let $\left(\sigma, B_{1}, B_{2}, \ldots, B_{k}, \bar{B}\right)$ be a non-crossing circular half-permutation of $[\mathrm{m}]$. We denote $x_{i}$ the initial point of $B_{i}$ relative to $\bar{B}$ and by $y_{i}$ the final point of $B_{i}$.

Remark 13. In the following theorem we have two non-crossing circular half-permutations each with $k$ open blocks. We show that one can construct $k$ non-crossing annular permutations from the pair of halfpermutations. The diagrammatic interpretation is very simple. We fix an open block on the first half-permutation and then there are $k$ open blocks on the second half-permutation to connect it to. Once this choice has been made there are no further choices for pairing up the remaining $k-1$ open blocks on each half-permutation.
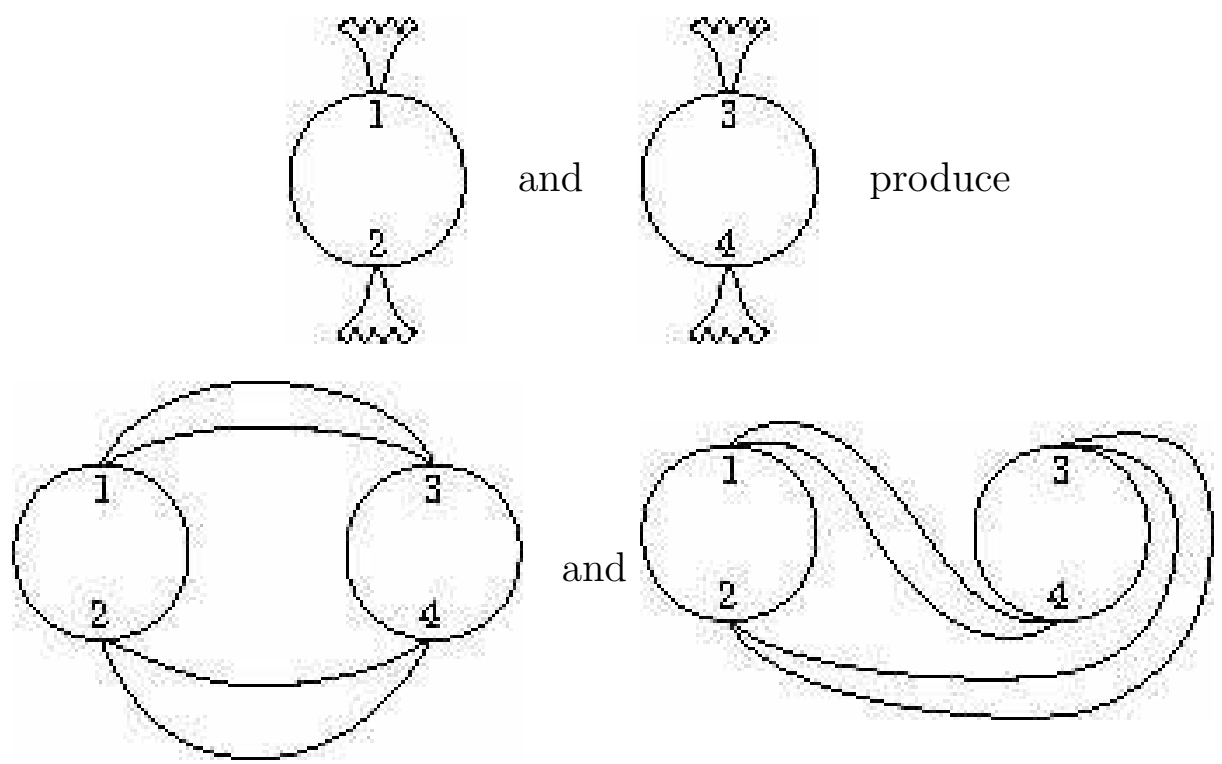

Theorem 14. Let $\left(\sigma, B_{1}, \ldots, B_{k}, \bar{B}\right)$ and $\left(\tau, C_{1}, \ldots, C_{k}, \bar{C}\right)$ be noncrossing circular half-permutations of $[m]$ and $[n]$ respectively, each with $k$ open blocks.

Then there are exactly $k$ non-crossing $(m, n)$-annular permutations $\pi^{(1)}, \pi^{(2)}, \ldots, \pi^{(k)}$ such that for each $i, \pi_{1}^{(i)}=\sigma$ and $\pi_{2}^{(i)}=\tau$. 
Proof. Let $1 \leq s \leq k$; we shall construct $\pi^{(s)}$. We shall regard $\tau$ as a non-crossing permutation of $[m+1, m+n]$ by identifying $[n]$ with its translate in $[m+1, m+n]$. Moreover shall denote by $\tilde{\gamma}_{n}$ the permutation of $[m+1, m+n]$ with the one cycle $(m+1, m+2, \ldots, m+n)$. Let the open blocks $B_{1}, B_{2} \ldots, B_{k}$ of $\sigma$ and the open blocks $C_{1}, C_{2}, \ldots, C_{k}$ of be arranged in cyclic order. Let $x_{1}, x_{2}, \ldots, x_{k}$ and $y_{1}, y_{2}, \ldots, y_{k}$ be such that $B_{i} \cap \bar{B}=\left\{x_{i}\right\}$ and $C_{i} \cap \bar{C}=\left\{y_{i}\right\}$. Let

$$
\pi^{(s)}=\left(x_{1}, y_{k-1+s}\right)\left(x_{2}, y_{k-2+s}\right) \cdots\left(x_{k}, y_{s}\right) \sigma \tau
$$

where the index of $y_{k-i+s}$ is interpreted modulo $k$. Let us show that $\#\left(\pi^{(s)}\right)+\#\left(\gamma_{m, n}\left(\pi^{(s)}\right)^{-1}\right)=m+n$. This will show that $\pi^{(s)}$ is a noncrossing annular permutation by the geodesic condition, [MN], Theorem 6.1 .

Let $\pi_{1}=\left(x_{1}, y_{k-1+s}\right) \sigma \tau$. Then $\#\left(\pi_{1}\right)=\#(\sigma)+\#(\tau)-1$ as $B_{1}$ and $C_{k-1+s}$ are disjoint cycles of $\sigma \tau$. Also

$\#\left(\gamma_{m, n} \pi_{1}^{-1}\right)=\#\left(\gamma_{m, n} \sigma^{-1} \tau^{-1}\left(x_{1} y_{k-1+s}\right)\right)=\#\left(\gamma_{m} \sigma^{-1}\right)+\#\left(\tilde{\gamma}_{n} \tau^{-1}\right)-1$

as $\bar{B}$ and $\bar{C}$ are disjoint cycles of $\gamma_{m, n} \sigma^{-1} \tau^{-1}$. Thus by the geodesic condition $\pi_{1}$ is non-crossing annular.

Now let $\pi_{r}=\left(x_{1}, y_{k-1+s}\right) \cdots\left(x_{r}, y_{k-r+s}\right) \sigma \tau$. We shall show by induction that $\pi_{r}$ is non-crossing annular. Since $B_{i}$ and $C_{k-i+s}$ are disjoint cycles of $\pi_{i-1}$ we have that $\#\left(\pi_{r}\right)=\#\left(\pi_{r-1}\right)-1$. We shall complete the proof by showing that $\#\left(\gamma_{m, n} \pi_{r}^{-1}\right)=\#\left(\gamma_{m, n} \pi_{r-1}^{-1}\right)+1$, as this will inductively show that $\pi_{r}$ satisfies the geodesic condition.

Write $\sigma=\sigma_{0} \bar{B}$ and $\tau=\tau_{0} \bar{C}$, then

$$
\gamma_{m, n} \pi_{r}^{-1}=\gamma_{m, n} \sigma_{0}^{-1} \tau_{0}^{-1}(\bar{B})^{-1}(\bar{C})^{-1}\left(x_{1}, y_{k-1+s}\right) \cdots\left(x_{r}, y_{k-r+s}\right)
$$

Thus it remains to shown that $x_{r}$ and $y_{k-r+s}$ are in the same cycle of

$$
B^{-1} C^{-1}\left(x_{1}, y_{k-1+s}\right) \cdots\left(x_{r-1}, y_{k-r+1+s}\right)
$$

or equivalently that $x_{r}$ and $x_{k-r+s}$ are in the same cycle of

$$
\left(x_{1}, y_{k-1+s}\right) \cdots\left(x_{r-1}, y_{k-r+1+s}\right) B C
$$

We establish this by recursively applying the following sublemma.

Sublemma Suppose $b_{1}<b_{2}<\cdots<b_{k}<c_{1}<c_{2}<\cdots<c_{l} ; 1 \leq$ $i_{1}<i_{2}<\cdots<i_{t} \leq k ; l \geq j_{1}>j_{2}>\cdots>j_{t} \geq 1$; and $D=$ $\left(b_{1}, b_{2}, \ldots, b_{k}, c_{1}, c_{2}, \ldots, c_{l}\right)$ be a cycle. For $r \neq s, b_{i_{s}}$ and $c_{j_{s}}$ are in the same cycle of $\left(b_{i_{r}}, c_{j_{r}}\right) D$.

Proof of sublemma By direct calculation,

$$
\left(b_{i_{r}}, c_{j_{r}}\right) D=\left(b_{1}, \ldots, b_{i_{r-1}}, c_{j_{r}}, \ldots, c_{l}\right)\left(b_{i_{r}}, \ldots, b_{k}, c_{1}, \ldots, c_{j_{r-1}}\right)
$$


If $s<r$ then $b_{i_{s}}$ and $c_{j_{s}}$ are in the first cycle; if $s>r$ they are in the second.

To complete the proof we must show that every non-crossing annular permutation arises from a pair of non-crossing circular half-permutations. So let $\pi$ be a non-crossing annular permutation. We must show that when we cut $\pi$ into $\pi_{1}$ and $\pi_{2}$, a pair of non-crossing circular halfpermutations, and then reassemble them we recover $\pi$. The only point that needs to be checked concerns the through blocks of $\pi$. So suppose that $D_{1}, D_{2}, \ldots, D_{k}$ are the through blocks of $\pi$ and we let $B_{1}=D_{1} \cap[m], B_{2}=D_{2} \cap[m], \ldots, B_{k}=D_{k} \cap[m]$ be the open blocks of $\pi_{1}$ and $C_{1}=D_{k} \cap[m+1, m+m], C_{2}=D_{k-1} \cap[m=1, m+n], \ldots$, $C_{k}=D_{1} \cap[m+1, m+n]$. Let us further suppose that the through blocks $D_{1}, \ldots, D_{k}$ have been ordered so that $B_{1}, \ldots, B_{k}$ are in cyclic order. By [MN], Def. 3.5 (AC-3) (see the middle diagram of [MN], Figure 3.7) the blocks $C_{k}, \ldots, C_{1}$ are in cyclic order. Let $x_{1}, \ldots, x_{k}$ be the initial points of $B_{1}, \ldots, B_{k}$; and similarly $y_{1}, \ldots, y_{k}$ the initial points of $C_{1}$, $\ldots C_{k}$. Then by [MN], Def. 3.5 (ANS-2), $D_{i}=\left(x_{i}, y_{k-i+1}\right) B_{i} C_{k-i+1}$ for $1 \leq i \leq k$. Thus $\pi=\left(x_{1}, y_{k}\right) \cdots\left(x_{k}, y_{1}\right) \pi_{1} \pi_{2}$.

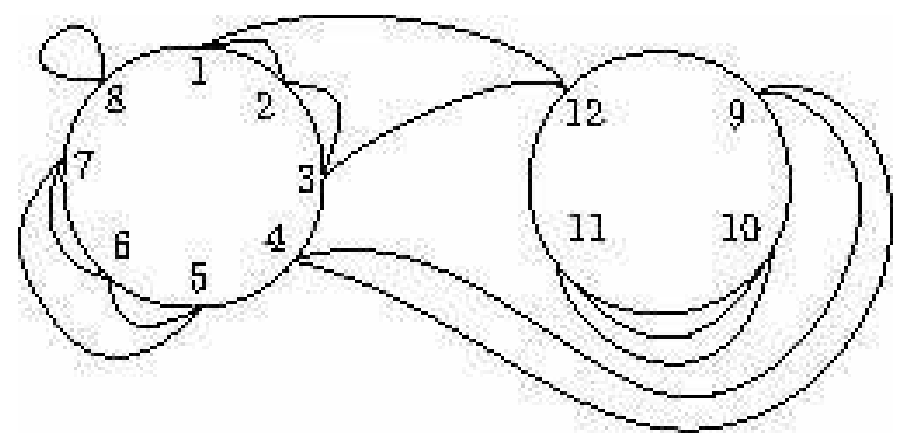

Figure 11. The two half-permutations of figure 9 reassembled to give the original permutation.

\section{Non-CROSSING Linear Half PERMUtations}

In the previous section we saw that a non-crossing annular permutation could be decomposed into two non-crossing circular halfpermutations. We shall need to decompose these circular half-permutations yet further into what we shall call non-crossing linear halfpermutations.

Suppose that $m=m_{1}+m_{2}+\ldots m_{k}$, with each $m_{i}>0$; and that we have intervals $I_{1}, \ldots, I_{k} \subset[m]$ of cyclically consecutive points. Moreover suppose that the interval $I_{j}$ has $m_{j}$ points each of colour $i_{j}$ and that cyclically adjacent intervals have different colours. 
Let $\left(\pi, B_{1}, \ldots, B_{k}, \bar{B}\right)$ be a non-crossing circular half-permutation of $[m]$ such that all points of a cycle have the same colour and that each interval $I_{r}$ meets at least one open block of $\pi$. Then each cycle of $\pi$ can meet only one interval and so for each $r,\left.\pi\right|_{I_{r}}$ is a non-crossing permutation of $I_{r}$. Moreover $\bar{B}$ meets the initial point of $I_{r}$. We shall formalize this notion in the definition below.

Definition 15. A non-crossing linear half-permutation of $[m]$ is a non-crossing circular half-permutation $\left(\pi, B_{1}, \ldots, B_{k}, \bar{B}\right)$ in which $1 \in$ $\bar{B}$. When $k=0$ we understand this to mean a non-crossing partition. We will denote by $N C L(m)_{k}$ the set of non-crossing linear halfpermutations on $[m]$ with $k$ open blocks.

If $I$ is a finite interval, a non-crossing linear half-permutation of $I$ is a non-crossing circular half-permutation $\left(\pi, B_{1}, \ldots, B_{k}, \bar{B}\right)$ in which the initial point of $I$ is in $\bar{B}$.

We summarize our discussion in the following theorem.

Theorem 16. Suppose $m=m_{1}+\cdots+m_{k}$ and we have intervals $I_{1}, \ldots, I_{k}$ of cyclically adjacent points with the interval $I_{r}$ of length $m_{r}$ and coloured $i_{r}$ and the colours $i_{1}, \ldots, i_{k}$ cyclically alternating. Let $\left(\pi, B_{1}, \ldots B_{k}, \bar{B}\right)$ be a non-crossing circular half-permutation of $[\mathrm{m}]$ where each interval $I_{r}$ meets one of the open blocks. Then each of $\left.\pi\right|_{I_{r}}$ is a non-crossing linear half-permutation whose open blocks are those of $\pi$ which meet $I_{r}$.

Given $0 \leq k \leq n$, let

$$
\bar{p}_{n, k}=\sum_{\pi \in N C L(n)_{k}} c^{\#(\pi)_{\mathrm{cl}}}
$$

where $\#(\pi)_{\mathrm{cl}}$ denotes the number of closed blocks of $\pi$. In this section we shall show that

$$
\bar{p}_{n+1, k}=\bar{p}_{n, k-1}+(1+c) \bar{p}_{n, k}+c \bar{p}_{n, k+1}
$$

This is the same recursion as for the $p_{n, k}$ 's (see equation (11)).

Theorem 17. For $0<k \leq n$

$$
\bar{p}_{n+1, k}=\bar{p}_{n, k-1}+(1+c) \bar{p}_{n, k}+c \bar{p}_{n, k+1}
$$

and for $k=0$

$$
\bar{p}_{n+1,0}=c \bar{p}_{n, 0}+c \bar{p}_{n, 1}
$$

Moreover $\bar{p}_{n, k}=p_{n, k}$ for $0 \leq k \leq n$. 
Proof. We must show that we can write $N C L(n+1)_{k}$ as a disjoint union of the four subsets

$$
N C L(n+1)_{k, 1}, N C L(n+1)_{k, 2}, N C L(n+1)_{k, 3}, N C L(n+1)_{k, 4}
$$

and exhibit bijections

$$
\begin{aligned}
& \phi_{1}: N C L(n+1)_{k, 1} \rightarrow N C L(n)_{k-1} \\
& \phi_{2}: N C L(n+1)_{k, 2} \rightarrow N C L(n)_{k} \\
& \phi_{3}: N C L(n+1)_{k, 3} \rightarrow N C L(n)_{k} \\
& \phi_{4}: N C L(n+1)_{k, 4} \rightarrow N C L(n)_{k+1}
\end{aligned}
$$

such that

$$
\begin{aligned}
\#\left(\phi_{i}(\pi)\right)_{\mathrm{cl}} & =\#(\pi)_{\mathrm{cl}} \text { for } i=1,2 \text { and } \\
1+\#\left(\phi_{i}(\pi)\right)_{\mathrm{cl}} & =\#(\pi)_{\mathrm{cl}} \text { for } i=3,4
\end{aligned}
$$

Given $\pi$ in $N C L(n+1)_{k}$ we look at the block containing $n+1$. There are four possibilities

- $n+1$ is in an open block which is a singleton;

- $n+1$ is in an open block which is not a singleton;

- $n+1$ is in a closed block which is a singleton; and

- $n+1$ is in a closed block which is not a singleton.

These four subsets clearly partition $N C L(n+1)_{k}$. Next we describe the maps $\left\{\phi_{1}, \phi_{2}, \phi_{3}, \phi_{4}\right\}$. In cases (1) and (3), $n+1$ is a singleton and $\phi_{1}$ and $\phi_{3}$ remove this singleton leaving the other blocks alone. In case (1) the number of open blocks decreases by one and the number of closed blocks is constant; so $\#\left(\phi_{1}(\pi)\right)_{\mathrm{cl}}=\#(\pi)_{\mathrm{cl}}$. In case $(3)$ the number of open blocks is unchanged but the number of closed blocks decreases by one so $1+\#\left(\phi_{3}(\pi)\right)_{\mathrm{cl}}=\#(\pi)_{\mathrm{cl}}$. In case $(2) n+1$ is part of an open block, we remove $n+1$ leaving the block open; thus both the number open and the number of closed blocks is unchanged.

For the case of (4) $n+1$ is part of a closed block. We remove $n+1$ and make the block open. Since $n+1$ is at an endpoint it cannot be covered by another block and thus we will not create a crossing by opening this block. However the number of open blocks increases by one and the number of closed blocks decreases by one and so $1+\#\left(\phi_{4}(\pi)\right)_{\mathrm{cl}}=\#(\pi)_{\mathrm{cl}}$.

It is easy to see that the maps in (1), (2), and (3) are bijections. In the case of (4) we just have to show that the construction can be reversed. Given a non-crossing linear half-permutation on $[n]$ with $k+1$ open blocks we add a point at $n+1$, make it part of the rightmost open block, and then make this block closed. 
In the case of $k=0$ we just have two cases (3) and (4) and we use the same maps as above.

We have now established that $\left\{\bar{p}_{n, k}\right\}_{n, k}$ satisfy the same recursion as $\left\{\bar{p}_{n, k}\right\}_{n, k}$, to prove that they are equal we have to show that they are equal for $n=1$.

By direct calculation we have $p_{1,0}=c$ and $p_{1,1}=1$. On the other hand when $n=1$ a partition can have only one block so $\bar{p}_{1,0}=c$ and $\bar{p}_{1,1}=1$.

We shall use the following theorem to calculate the limiting mean of $\mathcal{E}\left(\operatorname{Tr}\left(\Gamma_{n}\left(X_{N}\right)\right)\right)$ and $\mathcal{E}\left(\operatorname{Tr}\left(\Pi_{n}\left(X_{N}\right)\right)\right)$ as $N \rightarrow \infty$.

\section{Theorem 18.}

$$
\sum_{k=0}^{\left[\frac{n-1}{2}\right]} c^{k} p_{n, 2 k+1}=\sum_{\pi \in N C(n)} \#(\pi) c^{\#(\pi)-1}
$$

Proof. Let $\widetilde{N C}(n)$ be the set of pairs $(\pi, B)$ where $\pi \in N C(n)$ and $B$ is a block of $\pi$. Let $\psi(\pi, B)=c^{\#(\pi)-1}$. Then the right hand side can be written $\sum_{(\pi, B) \in \widetilde{N C}(n)} \psi(\pi, B)$.

Let us define a map

$$
\bigcup_{k=0}^{\left[\frac{n-1}{2}\right]} N C L(n)_{2 k+1} \rightarrow \widetilde{N C}(n)
$$

which we shall denote $\left(\pi, B_{1}, \ldots, B_{2 k+1}\right) \mapsto(\bar{\pi}, B)$ as follows. Arrange the open blocks $B_{1}, \ldots, B_{2 k+1}$ from left to right as follows. $\min \left(B_{1}\right)<$ $\min \left(B_{i}\right)$ for $i>1$ and $\max \left(B_{i}\right)<\max \left(B_{2 k+1}\right)$ for $i<2 k+1$. Then $\min \left(B_{2}\right)<\min \left(B_{i}\right)$ for $i>2$ and $\max \left(B_{i}\right)<\max \left(B_{2 k}\right)$ for $i<2 k$. Then we join $B_{1}$ with $B_{2 k+1}, B_{2}$ with $B_{2 k}, \ldots$, and $B_{k-1}$ with $B_{k+1}$. Call this new partition $\bar{\pi}$ and let $B=B_{k}$. As we joined these blocks from the outside in we have that $\bar{\pi}$ is non-crossing. Thus $(\bar{\pi}, B) \in$ $\widetilde{N C}(n)$.

Conversely starting with $(\bar{\pi}, B) \in \widetilde{N C}(n)$, let $k$ be the number of blocks covering $B$. We split each of these $k$ into two and declare them open. The open blocks of $\pi$ will be $B$ and these $2 k$ blocks.

If $\left(\pi, B_{1}, \ldots, B_{2 k+1}\right) \in N C L(n)_{2 k+1}$ with $j$ closed blocks then $k+$ $\#(\pi)_{\mathrm{cl}}=k+j$ and $\#(\bar{\pi})-1=\#(\pi)-k-1=k+j$, so $c^{k} c^{\#(\pi)_{\mathrm{cl}}}=$ $\psi(\bar{\pi}, B)$. Summing over $\cup_{k=0}^{\left[\frac{n-1}{2}\right]} N C L(n)_{2 k+1}$ now gives us the result. 
Remark 19. The equation

$$
P_{k}(z)=\left(\frac{P_{0}(z)-1}{c}\right)^{k} P_{0}(z)
$$

has the following combinatorial interpretation. Every non-crossing linear half-permutation with $k$ open blocks can be written as the concatenation of $k$ non-crossing linear half-permutations with one open block and one non-crossing linear half-permutation with zero open blocks. Indeed, given $\pi$ a non-crossing linear half-permutation with $k$ open blocks, let $\pi_{1}$ be all the blocks of $\pi$ starting from the left up to and including the first open block. Then $\pi_{2}$ starts after this open block and continues up to the second block, and so on until all the open blocks are exhausted; what remains is a non-crossing half-permutation with zero open blocks. We convert each of these $k$ half-permutations with one open block to a half-permutation with zero open blocks by closing the single open block. Note that this construction is reversible. Since we always require at least one block we subtract 1 from $P_{0}(z)$ and since we convert an open block to a closed block we divide by $c$.

\section{ReCURSion Formula FOR NON-CROSSING CIRCUlaR HALF-PERMUTATIONS}

In section 3 we constructed a family $\left\{\widetilde{\Gamma}_{n}\right\}_{n}$ of polynomials we called shifted Chebyshev polynomials of the first kind. In the notation established there $\widetilde{\Gamma}_{n}(x)=\sum_{k=0}^{n} g_{n, k}^{\prime} x^{k}$ where $g_{n, k}^{\prime} \in \mathbb{Z}[c]$. We established the recursion formula

$$
g_{n, k-1}^{\prime}=g_{n+1, k}^{\prime}+(1+c) g_{n, k}^{\prime}+c g_{n-1, k}^{\prime}
$$

for $n>1$ and

$$
g_{1, k-1}^{\prime}=g_{2, k}^{\prime}+(1+c) g_{1, k}^{\prime}+2 c g_{0, k}
$$

where $g_{n, k}^{\prime}=0$ whenever $n<0, k<0$ or $k>n$. We let $\widetilde{\Gamma}$ be the matrix with $(n, k)$ entry $g_{n, k}^{\prime}$ and $\left\{g_{n, k}\right\}_{n, k}$ be the entries of $\widetilde{\Gamma}^{-1}$. We showed that

$$
g_{n+1, k}=g_{n, k-1}+(1+c) g_{n, k}+c g_{n, k+1}
$$

and

$$
g_{n+1,0}=(1+c) g_{n, 0}+2 c g_{n, 1}
$$

We wish to show that these relations have an interpretation in terms of non-crossing circular half-permutations. Moreover this interpretation is essential for the proof of our main theorem (Theorem 1). 
Let $\pi$ be a non-crossing circular half-permutation on $[n]$ and $\#(\pi)_{\mathrm{cl}}$ the number of closed blocks. We wish to show that for $k \geq 1$ and all $n$

$$
g_{n, k}=\sum_{\pi} c^{\#(\pi)_{\mathrm{cl}}}
$$

where the sum is over all non-crossing circular half-permutations on $[n]$ with $k$ open blocks and $\#(\pi)_{\mathrm{cl}}$ is the number of closed blocks.

Let us denote be $N C C(n)$ the collection of non-crossing circular halfpermutations on $[n]$ and $N C C(n)_{k}$ the collection of non-crossing circular half-permutations on $[n]$ with $k$ open blocks.

In order to prove equation (16), even just for $k \geq 1$, we have to extend the definition of non-crossing circular half-permutations (Definition 3) to the case $k=0$.

Definition 20. A non-crossing circular half-permutation on $[n]$ with zero open blocks is a pair $(\pi, B)$ where $\pi$ is a non-crossing permutation on $[n]$ and $B$ is either a block of $\pi$ or a block of $\pi^{c}$. The block $B$ is called the designated block. The collection of non-crossing circular half-permutations with zero open blocks is denoted $N C C(n)_{0}$.

Notation 21. For all $n \geq 1$ and $n \geq k \geq 1$ let

$$
\bar{g}_{n, k}=\sum_{\pi \in N C C(n)_{k}} c^{\#(\pi)_{\mathrm{cl}}}
$$

and

$$
\bar{g}_{n, 0}=\sum_{\substack{(\pi, B) \in N C C(n)_{0} \\ B \in \pi^{c}}} c^{\#(\pi)}+\sum_{\substack{(\pi, B) \in N C C(n)_{0} \\ B \in \pi}} c^{\#(\pi)-1}
$$

For convenience we set $\bar{g}_{0,0}=1$.

Our goal in this section is to prove

Theorem 22. $\bar{g}_{n, k}=g_{n, k}$ for $0 \leq k \leq n$

We shall prove this theorem by showing that $\left\{\bar{g}_{n, k}\right\}_{n, k}$ satisfy the same recurrence relation as $\left\{g_{n, k}\right\}_{n, k}$, namely equations (5) and (6).

We will also need to extend the definition of initial and final points to all the blocks of a half-permutation. Recall that in Definition 5 we supposed that $\bar{B}$ was a block of $\pi^{c}$ and we defined for an open block $B$ of $\pi$ two points (which coincide if $B$ is a singleton) which we called the initial and final points. We wish to extend this to the other blocks of $\pi$ and to the blocks of a half-permutation with zero open blocks. In the following definition $\gamma$ is the permutation of $[n]$ with the one cycle $(1,2,3, \ldots, n)$. 
Definition 23. Let $\pi \in N C(n)$ be a non-crossing permutation of $n$ and $B$ a block of $\pi$.

a) Suppose $\bar{B}$ a block of $\pi^{c}$ and $B \cap \bar{B}=\varnothing$. Let $j$ be any point of $\bar{B}$ and let $k$ be such that $\gamma^{k}(j) \in B$ but $\gamma^{l}(j) \notin B$ for all $1 \leq l<k$. Then $i=\pi^{k}(j)$ is the initial point of $B$ (relative to $\bar{B}$ ) and $\pi^{-1}(i)$ is the final point of $B$ (relative to $\bar{B}$ ). By the non-crossing property of $\pi, i$ is independent of the choice of $j$.

b) Suppose $B_{1} \neq B$ is a block of $\pi$. We choose any $j \in B$ and find the first $k$ such that $\gamma^{k}(j) \in B_{1}$, i.e. $\gamma^{k}(j) \in B_{1}$ but $\gamma^{l}(j) \notin B_{1}$ for $1 \leq l<k$. Let $i=\gamma^{k}(j)$, we call $i$ the initial point of $B_{1}$ (relative to $B$ ) and $\pi^{-1}(i)$ the final point of $B_{1}$. Again $i$ is not affected by the choice of $j$.

Remark 24. If $\bar{B}$ is a block of $\pi^{c}$ and $B$ is a block of $\pi$ with $B \cap \bar{B} \neq \varnothing$, then Definition 5 and $(a)$ above coincide.

In order to prove Theorem 22 we introduce a new way of describing elements of $N C C(n)_{k}$ using a dot structure. By a dot structure we mean placing either black or white dots on each of $1,1^{\prime}, 2,2^{\prime}, \ldots, n, n^{\prime}$.

Notation 25. Let $D_{j, k, n}$ be the collection of dot structures on $1,1^{\prime}, 2,2^{\prime}$, $\ldots, n, n^{\prime}$ such that

- there are $j$ black dots on primed numbers and the remaining $(n-j)$ primed numbers have white dots;

- there are $(k+j)$ white dots on unprimed numbers and black dots on the remaining $n-(j+k)$ unprimed numbers.

Theorem 26. For $k>0$ there is a bijection between the subset $\{\pi \in$ $N C C(n)_{k} \mid \pi$ has $j$ closed blocks $\}$ and $D_{j, k, n}$.

Proof. Suppose we are given a dot structure. We place the numbers $1,1^{\prime}, 2,2^{\prime}, \ldots, n, n^{\prime}$ around the circle in a clockwise fashion.

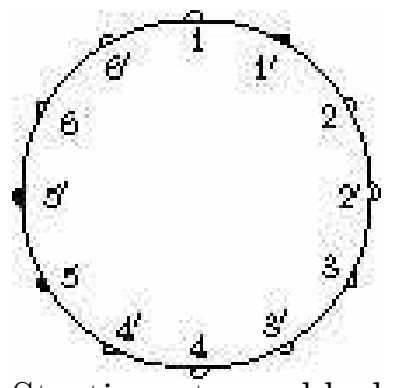

Figure 12. In this example $n=6$, $j=2$, and $k=3$. We place black dots on $1^{\prime}$ and $5^{\prime}$ and white dots on $2^{\prime}, 3^{\prime}, 4^{\prime}$ and $6^{\prime} . j+k=5$ so we place 5 white dots on a unprimed numbers, 1, 2, 3, 4, and 6 say. To complete we place a black dot on $5^{\prime}$.

Starting at any black dot we move in a counter-clockwise direction until we come to the first available white dot and connect these two dots. By available we mean that every time we pass over a black dot we must skip over an additional white dot. Since there are in total $n-k$ 
black dots and $n+k$ white dots, every black dot can be connected to a white dot in the manner described. Moreover since a black dot on a primed number can only be paired with a white dot on an unprimed number, there will be $k$ remaining white dots on unprimed numbers. Similarly a black dot on an unprimed number can only be paired with a white dot on a primed number so there will be $k$ white dots on primed numbers remaining.

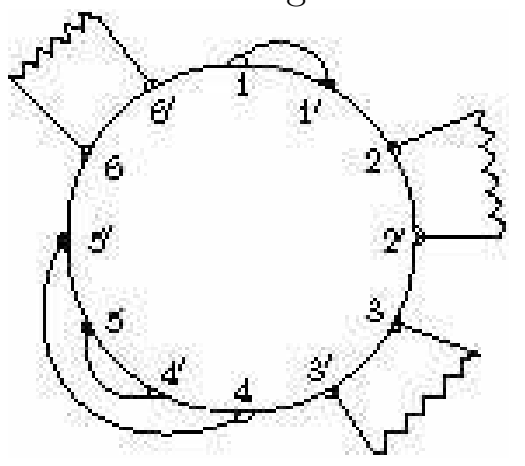

Figure 13. We then connect each black dot to its first available neighbour in the counter-clockwise direction. The $k$ remaining white dots on primed numbers get connected to the $k$ remaining white dots on unprimed numbers.

We connect each remaining white dot on a primed number to the first available white dot on an unprimed number, moving as before in the counter-clockwise direction.

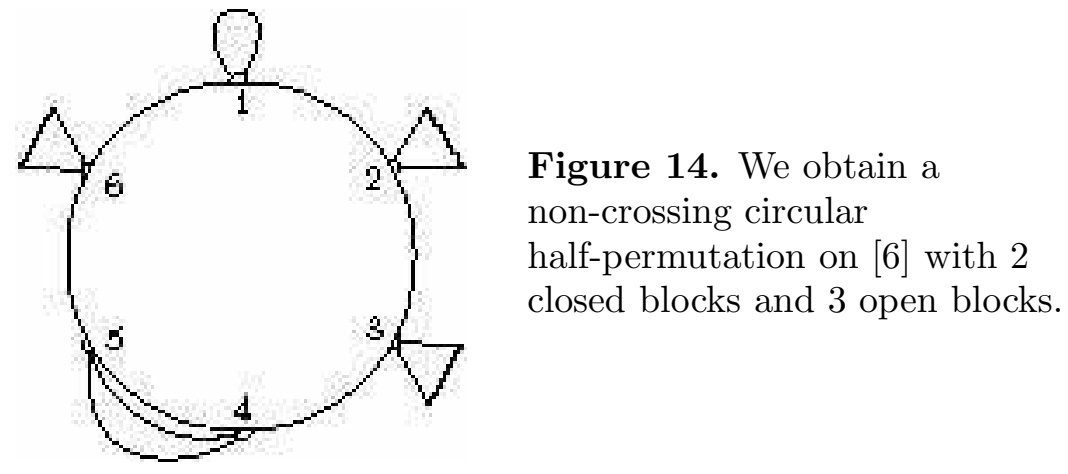

Finally we squeeze together each unprimed number $i$ with its corresponding primed number $i^{\prime}$ to form a non-crossing circular halfpermutation. Each black dot on a primed number starts a closed block. There are $n-j$ white dots on primed numbers of which $n-j-k$ are paired with black dots on unprimed numbers.

Each white dot on a primed number starts an open block and so we obtain $k$ open blocks and $j$ closed blocks. This incidentally shows that the number of such permutations is $\left(\begin{array}{c}n \\ j\end{array}\right)\left(\begin{array}{c}n \\ j+k\end{array}\right)$; so the coefficient of $c^{j}$ in

$$
\sum_{\pi \in N C C(n)_{k}} c^{\#(\pi)_{\mathrm{cl}}} \text { is }\left(\begin{array}{l}
n \\
j
\end{array}\right)\left(\begin{array}{c}
n \\
j+k
\end{array}\right) .
$$


This construction is reversible: starting with a non-crossing circular half-permutation with $k$ open blocks and $j$ closed blocks we arrange black and white dots as follows.

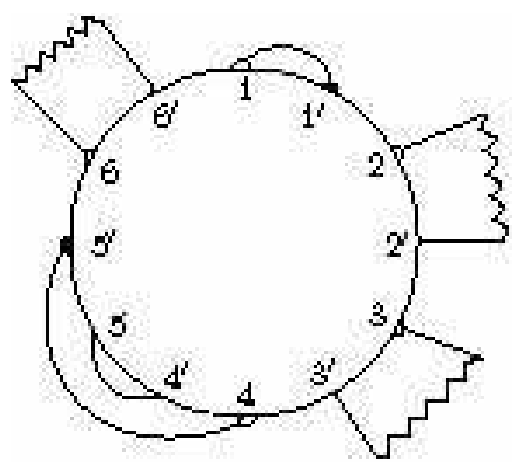

Figure 15. We start with a non-crossing circular half-permutation and we place a black dot on $1^{\prime}$ and $5^{\prime}$. We place white dots on $1,2,3,4$, and 6 .

On the unprimed numbers we place a white dot on the initial point of each block and black dots on the $n-j-k$ remaining points. On the primed numbers we place a black dot on the final point of each of the $j$ closed block and a white dot on each of the remaining points. This establishes a bijection between $N C C(n)_{k}$ and $D_{j, k, n}$.

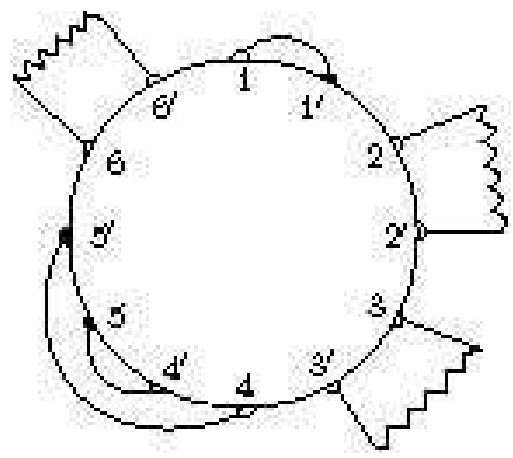

Figure 16. We then place a black dot on all the remaining unprimed numbers and a white dot on all the remaining primed numbers.

Theorem 27. There is a bijection between $D_{j, 0, n}$ and the subset of $N C C(n)_{0}:\left\{(\pi, B) \mid \pi\right.$ has $j$ blocks when $B \in \pi^{c}$, or $\pi$ has $j+1$ blocks when $B \in \pi\}$.

Proof. Let $(\pi, B) \in N C C(n)_{0}$ with $B \in \pi^{c}$. Suppose that $\pi$ has $j$ blocks, we shall construct a dot diagram in $D_{j, 0, n}$. We put a black dot on the prime of the final point of each block of $\pi$, all other primed numbers get a white dot. We put a white dot on the unprime of the initial point of each block of $\pi$; all other unprimes get a black dot. If a block of $\pi$ is a singleton then we put a black dot on the prime and a white dot on the unprime of the number. Thus there are $j$ black dots on primed numbers and $j$ white dots on unprimed numbers; so the dot diagram is in $D_{j, 0, n}$. 
Next suppose that $(\pi, B) \in N C C(n)_{0}$ with $j+1$ blocks and $B \in \pi$. We follow the same construction as before for each of the $j$ undesignated blocks. For the designated block $B$ we put a white dot on each unprimed number and a black dot on each primed number. Thus on the designated block there are no black dots on primed numbers or white dots on unprimed numbers. So again we obtain a dot pattern in $D_{j, 0, n}$.

Now suppose we have a dot pattern in $N C C(n)_{0}$ we must construct a non-crossing permutation $\pi$ and identify block $B$ either of $\pi$ or of $\pi^{c}$.

We obtain $\pi$ as in the proof of Theorem 26. We have $n$ black dots and $n$ white dots. We connect each black dot to the first available white dot. The blocks of $\pi$ are constructed as follows. Start at any of the $j$ white dots on an unprimed number and 'open' a block. We move in the clockwise direction adding points until we come to a black dot on a primed number, where we 'close' the block. If we encounter another white dot on an unprimed number before closing the block we open new block and close this one before closing the first one opened. These Catalan paths can only produce non-crossing permutations.

If this matching exhausts all of the points then we are in the case $B \in \pi^{c}$. We can identify the points of $B$ as follows. A point $i$ is in $B$ if it passes the following test. Starting at the unprime of $i$ and moving in a clockwise direction we count the number of white points passed minus the number of black points. If we can return to $i$ without the running total ever being negative, then $i \in B$.

If the matching doesn't exhaust all of the points then there are points remaining of the form black dot on unprimed number and white dot on unprimed number. These will then form the points of the designated block $B \in \pi$. As we can only encounter a black-white pair when there are no open blocks, $B$ cannot cross any of the previously constructed blocks. Thus $(\pi, B) \in N C C(n)_{0}$.

Theorem 28. For $k>0$

$$
\bar{g}_{n+1, k}=\bar{g}_{n, k-1}+(1+c) \bar{g}_{n, k}+c \bar{g}_{n, k+1}
$$

and for $k=0$

$$
\bar{g}_{n+1,0}=(1+c) \bar{g}_{n, 0}+2 c \bar{g}_{n, 1}
$$

Proof. For all $n$ and $k$ we write $N C C(n)_{k}$ as the disjoint union of four subsets according to the four possible dot patterns on $n$ :

$$
\begin{aligned}
& N C C(n)_{k, 1}=\left\{(\pi, B) \in N C C(n)_{k} \mid \text { white on } n \text { white on } n^{\prime}\right\} \\
& N C C(n)_{k, 2}=\left\{(\pi, B) \in N C C(n)_{k} \mid \text { black on } n \text { white on } n^{\prime}\right\} \\
& N C C(n)_{k, 3}=\left\{(\pi, B) \in N C C(n)_{k} \mid \text { white on } n \text { black on } n^{\prime}\right\}
\end{aligned}
$$




$$
N C C(n)_{k, 4}=\left\{(\pi, B) \in N C C(n)_{k} \mid \text { black on } n \text { black on } n^{\prime}\right\}
$$

We shall define maps

$$
\begin{aligned}
\phi_{1}: N C C(n+1)_{k, 1} \rightarrow N C C(n)_{k-1} \\
\phi_{2}: N C C(n+1)_{k, 2} \rightarrow N C C(n)_{k} \\
\phi_{3}: N C C(n+1)_{k, 3} \rightarrow N C C(n)_{k} \\
\phi_{4}: N C C(n+1)_{k, 4} \rightarrow N C C(n)_{k+1}
\end{aligned}
$$

such that $\#\left(\phi_{i}(\pi)\right)_{\mathrm{cl}}=\#(\pi)_{\mathrm{cl}}$ for $i=1,2$ and $\#\left(\phi_{i}(\pi)\right)_{\mathrm{cl}}=\#(\pi)_{\mathrm{cl}}-1$ for $i=3,4$.

On the level of dot diagrams the maps $\phi_{i}$ all simply delete the points $n+1,(n+1)^{\prime}$ and their dots except in the case $k=0$ and $i=1$. When $k=0$ and $i=1, \phi_{1}$ deletes the two white dots on $n+1$ and $(n+1)^{\prime}$ and then reverses the colour of all dots.

Let us check that each of the maps $\phi_{i}$ is a bijection and has the required effect on the number of closed blocks.

The case $i=1$. We remove a white dot on an unprimed number so the number of blocks decreases by one, however the number of black dots on primed numbers is unchanged so the block lost is a white block. Thus $\#\left(\phi_{1}(\pi)\right)_{\mathrm{cl}}=\#(\pi)_{\mathrm{cl}}$. Since we had $k$ open blocks to start with $\phi_{1}(\pi) \in N C C(n)_{k-1}$. The inverse map from $N C C(n)_{k-1}$ is to insert $n+1$ and $(n+1)^{\prime}$ and put white dots on them.

The case $i=2$. We remove a black dot on an unprimed number so the number of blocks is unchanged, moreover the number of black dots on primed numbers is unchanged so the number of closed blocks is unchanged. Thus $\#\left(\phi_{2}(\pi)\right)_{\mathrm{cl}}=\#(\pi)_{\mathrm{cl}}$. Since we had $k$ open blocks to start with $\phi_{2}(\pi) \in N C C(n)_{k}$. The inverse map from $N C C(n)_{k-1}$ is to insert $n+1$ and $(n+1)^{\prime}$ and put a black dot on $n+1$ and a white dot on $(n+1)^{\prime}$.

The case $i=3$. We remove a white dot on an unprimed number so the number of blocks is decreased by one, also the number of black dots on primed numbers is decreased by one so the number of closed blocks is decreased by one. Indeed, in this case we see that $n+1$ was a closed singleton and we removed it. Thus $\#\left(\phi_{3}(\pi)\right)_{\mathrm{cl}}=\#(\pi)_{\mathrm{cl}}-1$. Since we had $k$ open blocks to start with $\phi_{3}(\pi) \in N C C(n)_{k}$. The inverse map from $N C C(n)_{k-1}$ is to insert $n+1$ and $(n+1)^{\prime}$ and put a white dot on $n+1$ and a black dot on $(n+1)^{\prime}$.

The case $i=4$. We remove a black dot on an unprimed number so the number of blocks is unchanged, however the number of black dots 
on primed numbers is reduced by one so a closed block is lost and an open block is gained. Thus $\#\left(\phi_{4}(\pi)\right)_{\mathrm{cl}}=\#(\pi)_{\mathrm{cl}}-1$. Since we had $k$ open blocks to start with $\phi_{4}(\pi) \in N C C(n)_{k+1}$. The inverse map from $N C C(n)_{k+1}$ is to insert $n+1$ and $(n+1)^{\prime}$ and put black dots on them.

This construction works for all $n, k$, and $i$ except when $k=0$ and $i=1$. In this situation the number of black dots equals the number of white dots (because $k=0$ ) and we remove two white dots leaving two fewer white dots than black, which is forbidden. So in this case we remove the two white dots and change all black dots to white and all white to black. So when $k=0$ both $\phi_{1}$ and $\phi_{4}$ map into $N C C(n)_{1}$. This justifies the 2 in equation (18).

Theorem 29. For $n \geq 1$ and $k \geq 1$

$$
g_{n, k}=\sum_{\pi \in N C C(n)_{k}} c^{\#(\pi)_{\mathrm{cl}}}
$$

and

$$
g_{n, 0}=\sum_{\pi \in N C(n)} \#\left(\pi^{c}\right) c^{\#(\pi)}+\#(\pi) c^{\#(\pi)-1}
$$

Proof. We only have to check that we have equality of $\left\{g_{1,0}, g_{1,1}\right\}$ and $\left\{\bar{g}_{1,0}, \bar{g}_{1,1}\right\}$ so that the induction can start. We have by calculation $g_{1,0}=1+c$ and $g_{1,1}=1$. On the level of diagrams when $n=1$ we have one block with one element in it. When $k=0$ we can either choose $B$ to be this block or the single block in the complement so that $\bar{g}_{1,0}=1+c$. When $k=1$ we must have one open block and since there is only one block, zero closed blocks, thus $\bar{g}_{1,1}=1$.

Remark 30. There is an intriguing interpretation of equation (9) for non-crossing circular half-permutations analogous to that (remark 19) for non-crossing linear half-permutations.

$$
G_{n}(z)=\left(\frac{P_{0}(z)-1}{c}\right)^{n} G_{0}(z)
$$

Each non-crossing circular half-permutation with $k$ open blocks can be assembled from $k$ non-crossing linear half-permutations with one open block and one non-crossing circular half-permutation with zero open blocks. The point of insertion of linear permutations is determined by the designated block. We shall not present the details here.

\section{The Proof of Theorem 1}

Let for each $N, X_{N, 1}, \ldots X_{N, p}$ be $p$ independent $N \times N$ Wishart random matrices i.e. $X_{N, i}=G_{N, i}^{*} G_{N, i}$ with $G_{N, i}=\left\{g_{m, n}^{(N, i)}\right\}_{m, n}$ an $M \times N$ 
Gaussian random matrix with independent entries and $\mathcal{E}\left(\left|g_{m, n}^{(n, i)}\right|^{2}\right)=$ $1 / N$. Fix $k, l>1$ and let $\vec{m}=\left(m_{1}, \ldots, m_{k}\right)$ and $\vec{n}=\left(n_{1}, \ldots, n_{l}\right)$ with $m_{s}>0$ and $n_{t}>0$ for all $s$ and $t$. Suppose also that we have $i_{1}, \ldots i_{k}$ and $j_{1}, \ldots j_{l}$ with $1 \leq i_{s}, j_{t} \leq p$ which are each cyclically alternating i.e. for $\left|s_{1}-s_{2}\right|=1, i_{s_{1}} \neq i_{s_{2}}$ and for $\left|t_{1}-t_{2}\right|=1, j_{t_{1}} \neq j_{t_{2}}$ and in addition $i_{k} \neq i_{1}$ and $j_{l} \neq j_{1}$.

Let

$$
S_{\vec{m}, \vec{i}}=\operatorname{Tr}\left(\Pi_{m_{1}}\left(X_{N, i_{1}}\right) \cdots \Pi_{m_{k}}\left(X_{N, i_{k}}\right)\right)
$$

and

$$
S_{\vec{n}, \vec{j}}=\operatorname{Tr}\left(\Pi_{n_{1}}\left(X_{N, j_{1}}\right) \cdots \Pi_{n_{l}}\left(X_{N, j_{l}}\right)\right)
$$

From [MN] Theorem 7.5 we have.

\section{Theorem 31.}

$$
\lim _{N} \kappa_{2}\left(\operatorname{Tr}\left(X_{N, i_{1}}^{m_{1}} \cdots X_{N, i_{k}}^{m_{k}}\right), \operatorname{Tr}\left(X_{N, j_{1}}^{n_{1}} \cdots X_{N, j_{l}}^{n_{l}}\right)\right)=\left|S_{N C}(\vec{m}, \vec{n})\right|_{c}
$$

where the undefined notation is explained below.

Notation 32. Let $\vec{u}=\left(u_{1}, \ldots, u_{k}\right)$ and $\vec{v}=\left(v_{1}, \ldots, v_{l}\right)$ where $u_{1}, u_{2}$, $\ldots, u_{k}$ and $v_{1}, v_{2}, \ldots, v_{l}$ are positive integers. Let $u=u_{1}+u_{2}+\cdots+u_{k}$ and $v=v_{1}+v_{2}+\cdots+v_{l}$.

Let $S_{N C}(\vec{u}, \vec{v})$ be the set of non-crossing annular $(u, v)$-permutations where

- on the $u$-circle we have $k$ intervals $I_{1}, I_{2}, \ldots, I_{k}$ of points with $u_{s}$ points in the interval $I_{s}$ all of colour $i_{s}$;

- on the $v$-circle we have $l$ intervals $J_{1}, J_{2}, \ldots, J_{l}$ of points with $j_{t}$ points in the interval $J_{t}$ all of colour $j_{t}$;

- only points of the same colour can be connected.

To simplify the notation we have omitted the dependence on the colours $i_{1}, \ldots, i_{k}$ and $j_{1}, \ldots, j_{l}$; since we only work with one set of colours this should not cause any confusion.

Notation 33. For $\vec{x}=\left(x_{1}, \ldots, x_{k}\right)$ and $\vec{y}=\left(y_{1}, \ldots, y_{l}\right)$ let $S_{N C}\left(\begin{array}{ll}\vec{u} & \vec{v} \\ \vec{x} & \vec{y}\end{array}\right)$ $=S_{N C}\left(\begin{array}{c}u_{1} \\ x_{1}\end{array}, \ldots,{ }_{x_{k}}^{u_{k}} ; \begin{array}{c}v_{1} \\ y_{1}\end{array}, \ldots, \begin{array}{c}v_{l} \\ y_{l}\end{array}\right)$ be the permutations in $S_{N C}(\vec{u}, \vec{v})$ such that from the interval $I_{s}$ there are exactly $x_{s}$ blocks that meet the $v$ circle and from the interval of $J_{t}$ there are exactly $y_{t}$ blocks that meet the $u$-circle, i.e. $I_{s}$ has $x_{s}$ through-blocks and $J_{t}$ has $y_{t}$ through-blocks.

If $x_{s}=u_{s}$ then we shall write $S_{N C}\left(\begin{array}{l}u_{1} \\ x_{1}\end{array}, \ldots, \underline{u}_{s}, \ldots,{ }_{x_{k}}^{u_{k}} ;{ }_{y_{1}}^{v_{1}}, \ldots,{ }_{y_{l}}^{v_{l}}\right)$ in place of $S_{N C}\left(\begin{array}{l}u_{1} \\ x_{1}\end{array}, \ldots,{ }_{u_{s}}^{u_{s}}, \ldots,{ }_{x_{k}}^{u_{k}} ; v_{1}, \ldots, \begin{array}{l}v_{l} \\ y_{l}\end{array}\right)$. Note that the condition $x_{s}=u_{s}$ means that every point of $I_{s}$ is in a different block and each of these blocks is a through-block. 
If $\vec{x}=\vec{u}$ and $\vec{y}=\vec{v}$ we shall write $S_{N C}(\underline{\vec{u}}, \underline{\vec{v}})$ in place of $S_{N C}\left(\begin{array}{ll}\vec{u} & \vec{v} \\ \vec{u} & \vec{v}\end{array}\right)$. In this case every block is a pair connecting the two circles (i.e. a spoke diagram), respecting the colours.

Notation 34. Suppose $A \subset S_{N C}(m, n)$. Let $|A|_{c}=\sum_{\pi \in A} c^{\#(\pi)}$. Suppose $1 \leq s<t \leq k$. Let $N C([s, t])$ be the non-crossing partitions $\pi$ of $[s, t]=\{s, s+1, \ldots, t\}$ that respect the colouring given by $i_{s}, \ldots, i_{t}$, i.e. are such that if $a$ and $b$ are in the same block of $\pi$ then $i_{a}=i_{b}$.

Let $X_{s, t}=\cup_{s \leq r \leq t} I_{r}$ with the colourings given by $i_{s}, \ldots, i_{t}$, i.e. the points of $I_{r}$ are coloured $i_{r}$. Let $N C\left(X_{s, t}\right)$ be the non-crossing partitions of $X_{s, t}$ that respect the colouring, i.e. each block only contains one colour.

Given $\pi \in N C\left(X_{s, t}\right)$ we obtain a partition $\nu_{\pi} \in N C([s, t])$ in which $r_{1}, r_{2} \in[s, t]$ are in the same block whenever a block of $\pi$ meets both the intervals $I_{r_{1}}$ and $I_{r_{2}}$. The idea is to take a partition of $X_{s, t}$ that respects the colours and only remember how the partition connects the intervals, i.e. shrink each interval to a point.

For $B \subset[s, t]$ such that $i_{r_{1}}=i_{r_{2}}$ for all $r_{1}, r_{2} \in B$, let $X_{B}=\cup_{r \in B} I_{r}$. Let $\sigma_{B}$ be the partition of $X_{B}$ in which each interval $I_{r}(r \in B)$ is a block of $\sigma_{B}$.

Let

$$
p_{B}=\sum_{\substack{\pi \in N C\left(X_{B}\right) \\ \sigma_{B} \vee \pi=1_{X_{B}}}} c^{\#(\pi)}
$$

i.e the sum is over all $\pi$ that connect all the intervals $\left\{I_{r}\right\}_{r \in B}$.

Remark 35. (i) Suppose $s<t, x_{s}=\cdots=x_{t}=0, x_{s-1}>0, x_{t+1}>0$ and $i_{s-1} \neq i_{t+1}$. Then by Theorem [16,

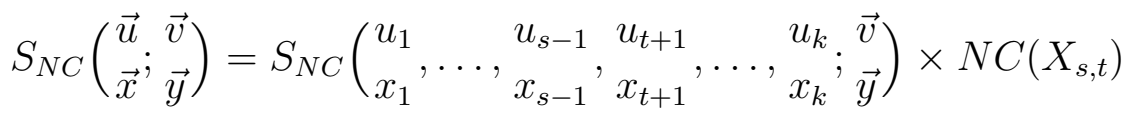

If $i_{s-1}=i_{t+1}$ then

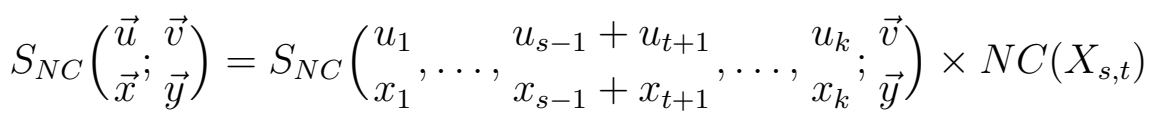

(ii) If $B=\{w\}$ is a singleton then

$$
p_{B}=\sum_{\pi \in N C\left(I_{w}\right)} c^{\#(\pi)}=p_{u_{w}, 0}
$$

Lemma 36.

$$
\left|N C\left(X_{s, t}\right)\right|_{c}=\sum_{\substack{\tau \in N C([s, t]) \\ \tau=\left\{B_{1}, \ldots, B_{q}\right\}}} \prod_{1 \leq i \leq q} p_{B_{i}}
$$


Proof. We partition $N C\left(X_{s, t}\right)$ over $N C([s, t])$, i.e. write

$$
N C\left(X_{s, t}\right)=\bigcup_{\tau \in N C([s, t])}\left\{\pi \in N C\left(X_{s, t}\right) \mid \nu_{\pi}=\tau\right\}
$$

where $\nu_{\pi}$ is the partition of the colours induced by $\pi$. Combine this with the observation that for $\tau=\left\{B_{1}, \ldots, B_{q}\right\}$

$$
\begin{gathered}
\left\{\pi \in N C\left(X_{s, t}\right) \mid \nu_{\pi}=\tau\right\}=\left\{\rho \in N C\left(X_{B_{1}}\right) \mid \rho \vee \sigma_{B_{1}}=1_{X_{B_{1}}}\right\} \\
\times \cdots \times\left\{\rho \in N C\left(X_{B_{q}}\right) \mid \rho \vee \sigma_{B_{q}}=1_{X_{B_{q}}}\right\}
\end{gathered}
$$

Lemma 37. Suppose that $x_{i}=0$ for some $i$. Then

$$
\sum_{\substack{u_{1}, \ldots, u_{k} \\
v_{1}, \ldots, v_{l}}} \prod_{\substack{1 \leq r \leq k \\
1 \leq s \leq l}} p_{m_{r}, u_{r}}^{\prime} p_{n_{s}, v_{s}}^{\prime}\left|S_{N C}\left(\begin{array}{cc}
\vec{m} & \vec{n} \\
\vec{x} & \vec{y}
\end{array}\right)\right|_{c}=0
$$

Proof. By invariance of the trace under cyclic permutatione we may suppose $x_{1}=\cdots=x_{t}=0$ and $x_{t+1}>0$. Furthermore we shall assume that $i_{t+1} \neq i_{k}$, the proof when $i_{t+1}=i_{k}$ is handled similarly.

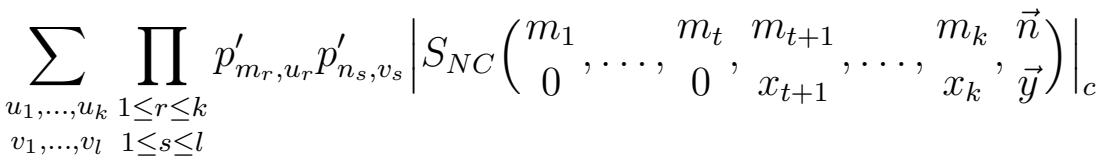

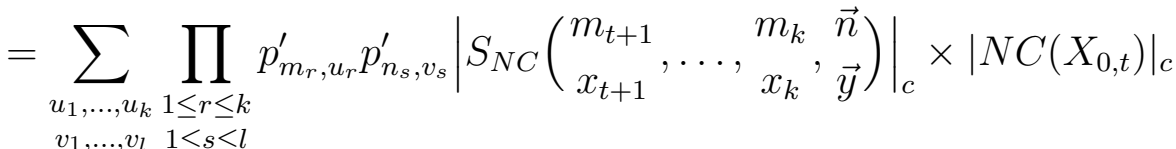

By Lemma 36] we can write $\left|N C\left(X_{0, t}\right)\right|_{c}$ as a sum over $N C([0, t])$. Since the colours are cyclically alternating we know that each $\tau$ in $N C([0, t])$ must contain at least one singleton $\{a(\tau)\}$ and by Remark 35] (ii) the corresponding $p_{B}$ is a $p_{u_{a(\tau)}, 0}$; we have that for some $M_{\tau}$

$$
\left|N C\left(X_{0, t}\right)\right|_{c}=\sum_{\tau \in N C([0, t])} p_{u_{a(\tau)}, 0} M_{\tau}
$$

Inserting this into our equation above we continue

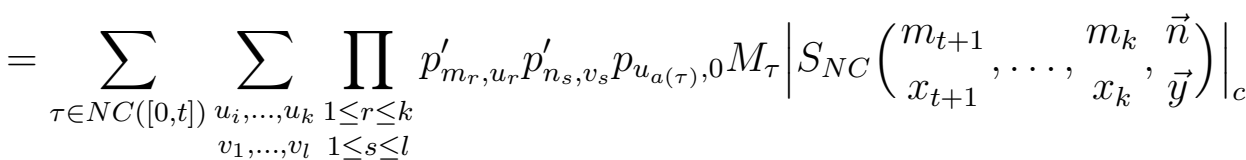

$$
\begin{aligned}
& =0
\end{aligned}
$$

Because, by Theorem [17. $\sum_{u_{a(\tau)}} p_{m_{a(\tau)}, u_{a(\tau)}}^{\prime} p_{u_{a(\tau)}, 0}=\delta_{m_{a(\tau), 0}}=0$. 


\section{Proposition 38.}

$$
\lim _{N \rightarrow \infty} \kappa_{2}\left(S_{\vec{m}, \vec{i}}, S_{\vec{n}, \vec{j}}\right)=\left|S_{N C}(\underline{\underline{m}}, \underline{\vec{n}})\right|_{c}
$$

Proof.

$$
\begin{aligned}
& \lim _{N} \kappa_{2}\left(S_{\vec{m}, \vec{i}}, S_{\vec{n}, \vec{j}}\right) \\
& =\lim _{N} \sum_{\substack{u_{1}, \ldots, u_{k} \\
v_{1}, \ldots, v_{l}}} \prod_{\substack{1 \leq r \leq k \\
1 \leq s \leq l}} p_{m_{r}, u_{r}}^{\prime} p_{n_{s}, v_{s}}^{\prime} \kappa_{2}\left(X_{N, i_{1}}^{u_{1}} \cdots X_{N, i_{k}}^{u_{k}}, X_{N, j_{1}}^{v_{1}} \cdots X_{N, j_{l}}^{v_{l}}\right) \\
& \stackrel{T h}{=} \sum_{\substack{31 \\
u_{1}, \ldots, u_{k} \\
v_{1}, \ldots, v_{l}}} \prod_{\substack{1 \leq r \leq k \\
1 \leq s \leq l}} p_{m_{r}, u_{r}}^{\prime} p_{n_{s}, v_{s}}^{\prime}\left|S_{N C}\left(u_{1}, \ldots, u_{k} ; v_{1}, \ldots, v_{l}\right)\right|_{c}
\end{aligned}
$$

we write $S_{N C}\left(u_{1}, \ldots, u_{k} ; v_{1}, \ldots, v_{l}\right)$ as a disjoint union over all $\vec{x}$ and $\vec{y}$ of $S_{N C}\left(\begin{array}{c}u_{1} \\ x_{1}\end{array}, \ldots, \begin{array}{l}u_{k} \\ x_{k}\end{array} ; \begin{array}{l}v_{1} \\ y_{1}\end{array}, \ldots, \begin{array}{l}v_{l} \\ y_{l}\end{array}\right)$

$$
=\sum_{\substack{u_{1}, \ldots, u_{k} \\
v_{1}, \ldots, v_{l}}} \prod_{\substack{1 \leq r \leq k \\
1 \leq s \leq l}} p_{m_{r}, u_{r}}^{\prime} p_{n_{s}, v_{s}}^{\prime} \sum_{\vec{x}, \vec{y}}\left|S_{N C}\left(\begin{array}{c}
u_{1} \\
x_{1}
\end{array}, \ldots, \begin{array}{c}
u_{k} \\
x_{k}
\end{array} \begin{array}{c}
v_{1} \\
y_{1}
\end{array}, \ldots, \begin{array}{c}
v_{l} \\
y_{l}
\end{array}\right)\right|_{c}
$$

by Lemma 37 we have,

$$
=\sum_{\substack{u_{1}, \ldots, u_{k} \\
v_{1}, \ldots, v_{l}}} \prod_{\substack{1 \leq r \leq k \\
1 \leq s \leq l}} p_{m_{r}, u_{r}}^{\prime} p_{n_{s}, v_{s}}^{\prime} \sum_{\substack{\vec{x}, \vec{y} \\
x_{1}, \ldots, x_{k}>0}}\left|S_{N C}\left(\begin{array}{c}
u_{1} \\
x_{1}
\end{array}, \ldots, \begin{array}{c}
u_{k} \\
x_{k} ; \\
y_{1}
\end{array}, \ldots, \begin{array}{c}
v_{l} \\
y_{l}
\end{array}\right)\right|_{c}
$$

by Lemma 37 again we have,

$$
=\sum_{\substack{u_{1}, \ldots, u_{k} \\
v_{1}, \ldots, v_{l}}} \prod_{\substack{1 \leq r \leq k \\
1 \leq s \leq l}} p_{m_{r}, u_{r}}^{\prime} p_{n_{s}, v_{s}}^{\prime} \sum_{\substack{x_{1}, \ldots, x_{k}>0 \\
y_{1}, \ldots, y_{l}>0}}\left|S_{N C}\left(\begin{array}{c}
u_{1} \\
x_{1}
\end{array}, \ldots, x_{k}^{u_{k}} ; y_{1}, \ldots, \begin{array}{c}
v_{l} \\
y_{l}
\end{array}\right)\right|_{c}
$$

Now we have for each interval at least one block passing to the opposite circle. Thus on each interval we have a non-crossing linear half-permutation. Thus

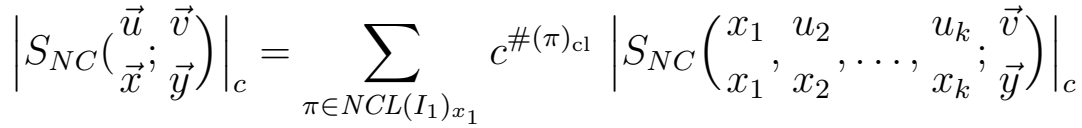

$$
\begin{aligned}
& =p_{u_{1}, x_{1}}\left|S_{N C}\left(\underline{x_{1}}, \begin{array}{l}
u_{2} \\
x_{2}
\end{array}, \ldots, \begin{array}{l}
u_{k} \\
x_{k}
\end{array} ; \begin{array}{l}
\vec{y} \\
\vec{y}
\end{array}\right)\right|_{c}
\end{aligned}
$$

by Theorem [17. We can repeat this for each $x_{i}$ and $y_{j}$ and thus obtain that our limit 


$$
\begin{aligned}
& =\sum_{\substack{x_{1}, \ldots, x_{k}>0 \\
y_{1}, \ldots, y_{l}>0}} \sum_{u_{1}, \ldots, u_{k}} \prod_{\substack{1 \leq r \leq k \\
v_{1}, \ldots, v_{l} \\
1 \leq s \leq l}} p_{m_{r}, u_{r}}^{\prime} p_{n_{s}, v_{s}}^{\prime} p_{u_{r}, x_{r}} p_{v_{s}, y_{s}}\left|S_{N C}(\underline{\vec{x}} ; \underline{\vec{y}})\right|_{c} \\
& =\sum_{\substack{x_{1}, \ldots, x_{k}>0 \\
y_{1}, \ldots, y_{l}>0}}\left|S_{N C}(\underline{\vec{x}} ; \underline{\vec{y}})\right|_{c} \prod_{\substack{1 \leq r \leq k \\
1 \leq s \leq l}} \delta_{m_{r}, x_{r}} \delta_{n_{s}, y_{s}} \\
& =\left|S_{N C}(\underline{\vec{m}} ; \underline{\vec{n}})\right|_{c}
\end{aligned}
$$

Proposition 39. Let $k>1,1 \leq i \leq p$ and $i_{1}, i_{2}, \ldots, i_{k}$ be cyclically alternating, and $n, m_{1}, \ldots, m_{k}>0$. Then

$$
\lim _{N} \kappa_{2}\left(S_{\vec{m}, \vec{i}}, \Gamma_{n}\left(X_{N, i}\right)\right)=0
$$

Proof. As in the proof of Proposition 38

$$
\begin{aligned}
\lim _{N} & \kappa_{2}\left(S_{N}, \Gamma_{n}\left(X_{N, i}\right)\right) \stackrel{T h m[1]}{=} \sum_{u_{1}, \ldots, u_{k}, v} \prod_{1 \leq r \leq k} p_{m_{r}, u_{r}}^{\prime} q_{n, v}^{\prime}\left|S_{N C}\left(u_{1}, \ldots, u_{k} ; v\right)\right|_{c} \\
& =\sum_{x_{1}, \ldots, x_{k}>0} \sum_{u_{1}, \ldots, u_{k}, v} \prod_{1 \leq r \leq k} p_{m_{r}, u_{r}}^{\prime} q_{n, v}^{\prime} \mid S_{N C}\left(\begin{array}{l}
\left.u_{1}, \ldots, u_{k} ; v\right)\left.\right|_{x_{1}}, \ldots \\
x_{k}
\end{array}\right. \\
& =\sum_{x_{1}, \ldots, x_{k}>0} \sum_{u_{1}, \ldots, u_{k}, v} \prod_{1 \leq r \leq k} p_{m_{r}, u_{r}}^{\prime} p_{u_{r}, x_{r}} q_{n, v}^{\prime}\left|S_{N C}(\underline{\vec{x}} ; v)\right|_{c} \\
& =\sum_{x_{1}, \ldots, x_{k}>0, v} \prod_{1 \leq r \leq k} \delta_{m_{r}, x_{r}} q_{n, v}^{\prime}\left|S_{N C}(\underline{\vec{x}} ; v)\right|_{c} \\
& =\sum_{1 \leq v \leq n} q_{n, v}^{\prime}\left|S_{N C}(\underline{\vec{m}} ; v)\right|_{c} \\
& =0
\end{aligned}
$$

because $S_{N C}(\underline{\underline{m}} ; v)$ is empty.

Proposition 40. Suppose $m, n>0$.

$$
\lim _{N} \kappa_{2}\left(\Gamma_{m}\left(X_{N, i}\right), \Gamma_{n}\left(X_{N, j}\right)\right)=\delta_{i, j} \delta_{m, n} m c^{m}
$$

Proof.

$$
\lim _{N} \kappa_{2}\left(\Gamma_{m}\left(X_{N, i}\right), \Gamma_{n}\left(X_{N, j}\right)\right)=\sum_{\substack{1 \leq u \leq m \\ 1 \leq v \leq n}} q_{m, u}^{\prime} q_{n, v}^{\prime} \lim _{N} \kappa_{2}\left(X_{N, i}^{u}, X_{N, j}^{v}\right)
$$

Now $\lim _{N} \kappa_{2}\left(X_{N, i}^{u}, X_{N, j}^{v}\right)=0$ unless $i=j$, so for the rest of the proof we shall assume that $i=j$. Moreover as $i=j, \lim _{N} \kappa_{2}\left(X_{N}^{u}, X_{N}^{v}\right)=$ 
$\left|S_{N C}(u ; v)\right|_{c}$. Thus

$$
\begin{aligned}
\lim _{N} \kappa_{2}\left(\Gamma_{m}\left(X_{N}\right), \Gamma_{n}\left(X_{N}\right)\right) & \stackrel{T h m}{=} \sum_{\substack{1 \leq u \leq m \\
1 \leq v \leq n}} q_{m, u}^{\prime} q_{n, v}^{\prime} \sum_{\substack{0<x \leq u \\
0<y \leq v}}\left|S_{N C}\left(\begin{array}{ll}
u & v \\
x & y
\end{array}\right)\right|_{c} \\
& \stackrel{T h m}{=} \sum_{\substack{29 \\
0<x \leq u \leq u \leq m \\
0<y \leq v}} \sum_{\substack{1 \leq v \leq n \\
1 \leq u, u}}^{\prime} q_{n, v}^{\prime} q_{u, x} q_{v, y}\left|S_{N C}(\underline{x}, \underline{y})\right|_{c} \\
& =\sum_{\substack{0<x \leq u \\
0<y \leq v}} \delta_{m, x} \delta_{n, y}\left|S_{N C}(\underline{x}, \underline{y})\right|_{c} \\
& =\left|S_{N C}(\underline{m}, \underline{n})\right|_{c}
\end{aligned}
$$

Proof of Theorem 1. Given that all the cumulants of degree higher than three for words in $X_{N, 1}, \ldots, X_{N, k}$ asymptotically vanish, in order to prove asymptotic independence we only need calculate the first and second cumulants.

From Proposition 38 we see that $S_{\vec{m}, \vec{\imath}}$ and $S_{\vec{n}, \vec{\jmath}}$ are asymptotically independent unless $(\vec{m}, \vec{\imath})$ and $(\vec{n}, \vec{\jmath})$ are cyclically equivalent; and that the complex variance of $S_{\vec{m}, \vec{\imath}}$ is $|(\vec{m}, \vec{\imath})|$. From Proposition 39 we see that $\left\{\operatorname{Tr}\left(\Gamma_{n}\left(X_{N, i}\right)\right)\right\}_{n, i}$ are asymptotically independent from $\left\{S_{\vec{m}, \vec{\imath}}\right\}_{\vec{m}, \vec{\imath}}$. By Proposition 40 we see that $\operatorname{Tr}\left(\Gamma_{m}\left(X_{N, i}\right)\right)$ is asymptotically independent from $\operatorname{Tr}\left(\Gamma_{n}\left(X_{N, j}\right)\right)$ unless $(m, i)=(n, j)$ and that the variance of $\operatorname{Tr}\left(\Gamma_{m}\left(X_{N, i}\right)\right)$ converges to $m c^{m}$. What remains is to calculate the asymptotic moments of $\operatorname{Tr}\left(\Gamma_{n}\left(X_{N}\right)\right), \operatorname{Tr}\left(\Pi_{n}\left(X_{N}\right)\right)$, and $S_{\vec{m}, \vec{\imath}}$.

We start with $\lim _{N} \operatorname{Tr}\left(\Pi_{n}\left(X_{N}\right)\right)$. Since

$$
0=\int_{a}^{b} \Pi_{n}(t) d \mu_{c}(t)=\sum_{k=0}^{n} p_{n, k}^{\prime} \sum_{\pi \in N C(k)} c^{\#(\pi)}
$$

we have

$$
\begin{aligned}
\lim _{N} \mathcal{E}\left(\operatorname{Tr}\left(\Pi_{n}\left(X_{N}\right)\right)\right) & =\sum_{k=0}^{n} p_{n, k}^{\prime} \mathcal{E}\left(\operatorname{Tr}\left(X_{N}^{k}\right)\right) \\
& =\lim _{N} \sum_{k=0}^{n} p_{n, k}^{\prime} \mathcal{E}\left(\operatorname{Tr}\left(X_{N}^{k}-\sum_{\pi \in N C(k)} c^{\#(\pi)} I_{N}\right)\right) \\
& =\sum_{k=0}^{n} p_{n, k}^{\prime} \sum_{\pi \in N C(k)} c^{\prime} \#(\pi) c^{\#(\pi)-1}
\end{aligned}
$$


Where the last equality is from $[\mathrm{MN}]$, Cor. 9.4. Hence we must show that

$$
\sum_{k=0}^{n} p_{n, k}^{\prime} \sum_{\pi \in N C(k)} \#(\pi) c^{\#(\pi)-1}= \begin{cases}0 & n \text { even } \\ c^{m} & n=2 m+1\end{cases}
$$

In terms of our matrix $\Pi$ our claim is that

$$
\Pi\left(\begin{array}{c}
0 \\
1 \\
1+2 c \\
\vdots \\
\sum_{\pi} \#(\pi) c^{\#(\pi)-1} \\
\vdots
\end{array}\right)=\left(\begin{array}{c}
0 \\
1 \\
0 \\
c \\
0 \\
c^{2} \\
\vdots
\end{array}\right)
$$

Or by taking inverses we must show that

$$
\sum_{k=0}^{\left[\frac{n-1}{2}\right]} p_{n, 2 k+1} c^{k}=\sum_{\pi \in N C(n)} \#(\pi) c^{\#(\pi)-1}
$$

However this is precisely Theorem [18. Thus we have that

$$
\lim _{N} \mathcal{E}\left(\operatorname{Tr}\left(\Pi_{n}\left(X_{N}\right)-c \Pi_{n-2}\left(X_{N}\right)\right)\right)=0
$$

and thus by equation (14)

$$
\lim _{N} \mathcal{E}\left(\operatorname{Tr}\left(\Gamma_{n}\left(X_{N}\right)+\Gamma_{n-1}\left(X_{N}\right)\right)\right)=0
$$

Thus to prove that $\lim _{N} \mathcal{E}\left(\operatorname{Tr}\left(\Gamma_{n}\left(X_{N}\right)\right)=(-1)^{n} c^{\prime}\right.$ we check the first few values of $n$ by direct calculation $(n=0,1,2)$ and then obtain the rest by induction using the equation above $(n>2)$.

Finally we check that $S_{\vec{m}, \vec{\imath}}$ is asymptotically centered. Recall that $p_{k, 0}=\sum_{\pi \in N C(k)} c^{\#(\pi)}$ is the limit of $\left.\mathcal{E}\left(\operatorname{Tr}\left(X_{N}^{k}\right)\right)\right)$. It is enough to show that

$$
\lim _{N} \mathcal{E}\left(\operatorname{Tr}\left(\left(X_{N, i_{1}}^{m_{1}}-p_{m_{1}, 0} I_{N}\right) \cdots\left(X_{N, i_{k}}^{m_{k}}-p_{m_{k}, 0} I_{N}\right)\right)\right)=0
$$

and then take linear combinations.

Recall that $\mathcal{E}\left(\operatorname{Tr}\left(X_{N}^{m}\right)\right)=N \sum_{\pi \in N C(m)}\left(\frac{M}{N}\right)^{\#(\pi)}+O\left(N^{-1}\right)$, so it suffices to keep track only of the terms of order $N$. Thus

$$
\begin{gathered}
\lim _{N} \mathcal{E}\left(\operatorname{Tr}\left(\left(X_{N, i_{1}}^{m 1}-p_{m_{1}, 0} I_{N}\right) \cdots\left(X_{N, i_{k}}^{m k}-p_{m_{k}, 0} I_{N}\right)\right)\right) \\
=\lim _{N} \sum_{A \subset[k]} \prod_{j \in A}\left(-p_{m_{j}, 0}\right) \mathcal{E}\left(\operatorname{Tr}\left(\prod_{j \notin A} X_{N, i_{j}}^{m_{j}}\right)\right)
\end{gathered}
$$




$$
=\lim _{N} N \sum_{A \subset[k]} \prod_{j \in A}\left(-p_{m_{j}, 0}\right) \sum_{\pi \in N C\left(A^{c}\right)}\left(\frac{M}{N}\right)^{\#(\pi)}
$$

were $N C(A)$ is the set of non-crossing partitions of $\cup_{j \in A}\left[m_{j-1}+1, m_{j}\right]$ that respect the colouring $\vec{i}$, i.e. $r$ and $s$ are in the same block of a partition only if $i_{r}=i_{s}$.

Now let us write $N C(A)$ as the disjoint union of the two subsets $N C(A)_{d}$ and $N C(A)_{c}$; where $N C(A)_{d}$ ( $d$ for disconnected) is the set of partitions on $N C(A)$ for which each block is a subset of an interval $\left[m_{j-1}+1, m_{j}\right]$ for some $j$, and $N C(A)_{c}$ is the set of partitions in $N C(A)$ that connect at least one pair of intervals. Note that $N C(A)_{d}=\prod_{j \in A} N C\left(\left[m_{j-1}+1, m_{j}\right]\right)$. Let us introduce an additional piece of notation. For a subset $C \subset[k]$ let $N C(C)_{c c}$ be the set of non-crossing partitions $\tau$ of $\cup_{j \in C}\left[m_{j-1}+1, m_{j}\right]$ such that every interval $I_{r}=\left[m_{r-1}+1, m_{r}\right]$ is connected to some other interval $I_{s}=\left[m_{s-1}+1, m_{s}\right]$ by some block of $\tau$. Thus

$$
N C\left(A^{c}\right)_{c}=\bigcup_{\substack{B \cup C=A^{c} \\ B \cap C=\varnothing}} N C(B)_{d} \times N C(C)_{c c}
$$

Note that for every $C \subset[k]$ for which $N C(C)_{c c}$ is not empty we must have $\left|C^{c}\right| \geq 2$ as every non-crossing partition of $k$ cyclically alternating colours must have at least two singletons.

Hence, suppressing an $O\left(N^{-1}\right)$ term, we have

$$
\begin{aligned}
\mathcal{E}( & \left.\operatorname{Tr}\left(\left(X_{N, i_{1}}^{m_{1}}-p_{m_{1}, 0} I_{N}\right) \cdots\left(X_{N, i_{k}}^{m_{k}}-p_{m_{k}, 0} I_{N}\right)\right)\right) \\
& =N \sum_{A \subset[k]} \prod_{j \in A}\left(-p_{m_{j}, 0}\right) \sum_{\substack{B \cup C=A^{c} \\
B \cap C=\varnothing}} \sum_{\substack{B \cap N C(B)_{d} \\
N}}\left(\frac{M}{N}\right)^{\#(\pi)} \sum_{\sigma \in N C(C)_{c c}}\left(\frac{M}{N}\right)^{\#(\sigma)} \\
& =N \sum_{C \subset[k]} \sum_{\sigma \in N C(C)_{c c}}\left(\frac{M}{N}\right)^{\#(\sigma)} \sum_{\substack{A \cup B=C^{c} \\
A \cap B=\varnothing}} \prod_{j \in A}\left(-p_{m_{j}, 0}\right) \prod_{l \in B} \sum_{\sigma \in N C\left(I_{l}\right)}\left(\frac{M}{N}\right)^{\#(\sigma)} \\
& =N \sum_{C \subset[k]} \sum_{\sigma \in N C(C)_{c c}}\left(\frac{M}{N}\right)^{\#(\sigma)} \prod_{j \in C^{c}}\left(\sum_{\pi \in N C\left(I_{j}\right)}\left(\frac{M}{N}\right)^{\#(\pi)}-p_{m_{j}, 0}\right)
\end{aligned}
$$

Since every $C$ for which $N C(C)_{c c}$ is not empty we have $\left|C^{c}\right| \geq 2$ there are always at least two factors in the product in the last expression above. The first factor of the form

$$
\sum_{\pi \in N C(I)}\left(\frac{M}{N}\right)^{\#(\pi)}-c^{\#(\pi)}
$$


absorbs the $N$ the second converges to 0 ; thus each term in the sum above converges to 0 and thus the $S_{\vec{m}, \vec{\imath}}$ s are centered.

\section{Wick Products}

In this section we show how to use non-crossing linear half-permutations to obtain relations for the Wick products of compound Poisson elements. These non-commutative polynomials were called the free Kailath-Segall polynomials by M. Anshelevich [A], §3.7, which are in turn a special case of the $q$-Kailath-Segall polynomials [A, §4.4]. In Theorem 43 we show how to expand a monomial as a sum of Wick polynomials. This is a special case of [A], Th. 4.11 (a). We define the convolution of a pair of non-crossing linear half-permutations and show in Theorem 47 that this corresponds to the product of two Wick products. This is a special case of [A] Thm. 4.11 (c).

We shall use the notation and definitions of [MS], $\S 4.2$. Let $\mathcal{D}$ be a unital $*$-algebra equipped with a tracial state $\psi$ and represent $\mathcal{D}$, via the GNS-representation, on $\mathcal{H}:=\overline{\mathcal{D}}^{\langle\cdot \cdot \cdot\rangle}$, where the inner product on $\mathcal{H}$ is given by

$$
\left\langle d_{1}, d_{2}\right\rangle:=\psi\left(d_{2}^{*} d_{1}\right)
$$

Let $\mathcal{F}(\mathcal{H})$ be the full Fock space over $\mathcal{H}$ with vacuum state $\Omega$ and for $d \in \mathcal{D}$ we have the following operators on $\mathcal{F}(\mathcal{H}): l(d)$ is the left creation operator, $l^{*}(d)$ is the left annihilation operator, $\Lambda(d)\left(d_{1} \otimes \cdots \otimes d_{n}\right)=$ $\left(d d_{1}\right) \otimes d_{2} \otimes \cdots d_{n}$ is the preservation operator (with $\Lambda(d) \Omega=0$ ), and $p(d)=l(d)+l^{*}\left(d^{*}\right)+\Lambda(d)+\psi(d) 1 . \Omega$ is a cyclic and separating vector for the algebra generated by $\{p(d) \mid d \in \mathcal{D}\}$. Thus for each $d_{1} \otimes \cdots \otimes d_{n} \in \mathcal{F}(\mathcal{H})$ there is a unique polynomial $W\left(d_{1} \otimes \cdots \otimes d_{n}\right)$ in the non-commuting variables $\{p(d) \mid d \in \mathcal{D}\}$ such that $W\left(d_{1} \otimes \cdots \otimes d_{n}\right) \Omega=$ $d_{1} \otimes \cdots \otimes d_{n}$. These are the Wick products.

One checks that $W(d)=p(d)-\psi(d)$ and from the definition of $p(d)$ on has immediately that

$$
\begin{aligned}
& W\left(d \otimes d_{1} \otimes \cdots \otimes d_{n}\right)=p(d) W\left(d_{1} \otimes \cdots \otimes d_{n}\right) \\
& -\psi\left(d d_{1}\right) W\left(d_{2} \otimes \cdots \otimes d_{n}\right)-W\left(\left(d d_{1}\right) \otimes \cdots \otimes d_{n}\right) \\
& -\psi(d) W\left(d_{1} \otimes d_{2} \otimes \cdots \otimes d_{n}\right)
\end{aligned}
$$

Our first goal in this section is to prove the following

$$
W\left(d_{1} \otimes \cdots \otimes d_{n}\right)^{*}=W\left(d_{n}^{*} \otimes \cdots \otimes d_{1}^{*}\right)
$$

In the course of proving this we shall extend the definition of Wick products to $W_{\pi}\left(d_{1} \otimes \cdots \otimes d_{n}\right)$ where $\pi$ is a non-crossing linear halfpermutation on $[n]$. When $\pi$ is the half-permutation in which each block is an open singleton $W_{\pi}\left(d_{1} \otimes \cdots \otimes d_{n}\right)=W\left(d_{1} \otimes \cdots \otimes d_{n}\right)$. 
Definition 41. Let $\left(\pi, B_{1}, \ldots, B_{k}\right)$ be a non-crossing linear half-permutation on $[k]$ with open blocks $B_{1}, \ldots, B_{k}$ and closed blocks $C_{1}, \ldots, C_{l}$. For a cycle $C=\left(r_{1}, \ldots, r_{u}\right)$ let $\psi_{C}\left(d_{1}, \ldots, d_{n}\right)=\psi\left(d_{r_{1}} \cdots d_{r_{u}}\right)$. Write each cycle $B_{j}=\left(r_{j, 1}, \ldots r_{j, v(j)}\right)$. Then we define

$$
\begin{aligned}
W_{\pi}\left(d_{1} \otimes \cdots \otimes d_{n}\right)= & \psi_{C_{1}}\left(d_{1}, \ldots, d_{n}\right) \cdots \psi_{C_{l}}\left(d_{1}, \ldots, d_{n}\right) \\
& \times W\left(\left(d_{r_{1,1}} \cdots d_{r_{1, v(1)}}\right) \otimes \cdots \otimes\left(d_{r_{k, 1}} \cdots d_{r_{k, v(k)}}\right)\right)
\end{aligned}
$$

Finally suppose that $S$ is a finite set of non-crossing linear half-permutations. Let

$$
W_{S}\left(d_{1} \otimes \cdots \otimes d_{n}\right)=\sum_{\sigma \in S} W_{\sigma}\left(d_{1} \otimes \cdots \otimes d_{n}\right)
$$

Example 42. Let $\pi=(1,2)(3)(4)(5,6)$ with $(1,2)$ and (4) open blocks and $(3)$ and $(5,6)$ closed. Then

$$
W_{\pi}\left(d_{1} \otimes \cdots \otimes d_{6}\right)=\psi\left(d_{3}\right) \psi\left(d_{5} d_{6}\right) W\left(\left(d_{1} d_{2}\right) \otimes d_{4}\right)
$$

Theorem 43. Let $d_{1}, d_{2}, \ldots d_{n} \in D$ and $\pi \in N C L(n)$. Then

a) for $d_{0} \in \mathcal{D}$

$$
\begin{aligned}
p\left(d_{0}\right) W_{\pi}\left(d_{1} \otimes \cdots \otimes d_{n}\right)=W_{\dot{\pi}}\left(d_{0} \otimes d_{1} \otimes \cdots \otimes d_{n}\right)+W_{\ddot{\pi}}\left(d_{0} \otimes d_{1} \otimes \cdots \otimes d_{n}\right) \\
+W_{\dot{\pi}}\left(d_{0} \otimes d_{1} \otimes \cdots \otimes d_{n}\right)+W_{\ddot{\pi}}\left(d_{0} \otimes d_{1} \otimes \cdots \otimes d_{n}\right)
\end{aligned}
$$

where the half-permutations $\dot{\pi}, \ddot{\pi}, \dot{\pi}, \ddot{\pi}$ of $\{0\} \cup[n]$ are obtained as follows:

- $\dot{\pi}$ : by adding an open singleton at 0;

- $\ddot{\pi}$ : by joining 0 to the first open block and then making this a closed block;

- $\dot{\pi}$ : by joining 0 to the first open block and leaving it open;

- $\ddot{\pi}$ : by making 0 a closed singleton.

b) $p\left(d_{1}\right) \cdots p\left(d_{n}\right)=\sum_{\pi \in N C L(n)} W_{\pi}\left(d_{1} \otimes \cdots \otimes d_{n}\right)$;

Proof. (a) follows from equation (19) when we rewrite it as

$$
\begin{aligned}
p\left(d_{0}\right) W\left(d_{1} \otimes \cdots \otimes d_{n}\right)=W\left(d_{0} \otimes d_{1} \otimes \cdots \otimes d_{n}\right) & \\
+\psi\left(d_{0} d_{1}\right) W\left(d_{2} \otimes \cdots \otimes d_{n}\right) & +W\left(\left(d_{0} d_{1}\right) \otimes \cdots \otimes d_{n}\right) \\
& +\psi\left(d_{0}\right) W\left(d_{1} \otimes d_{2} \otimes \cdots \otimes d_{n}\right)
\end{aligned}
$$

(b) follows by induction and the fact that every $\sigma \in N C L(\{0\} \cup[n])$ is $\dot{\pi}, \ddot{\pi}$, $\dot{\pi}$, or $\ddot{\pi}$ for exactly one $\pi \in N C L([n])$.

Corollary 44. For $d_{1} \ldots, d_{n} \in D$,

$$
W\left(d_{1} \otimes \cdots \otimes d_{n}\right)^{*}=W\left(d_{n}^{*} \otimes \cdots \otimes d_{1}^{*}\right)
$$


Proof. Take adjoints of equation $(b)$ in Theorem 43 and use induction and the fact that $N C L(n)$ is invariant under the reflection $i \mapsto n+1-i$.

Notation 45. Let $\pi \in N C L(m)$ and $\sigma \in N C L(n)$ be non-crossing linear half-permutations. Let the open blocks of $\pi$ be $B_{1}, \ldots, B_{j}$ and the open blocks of $\sigma$ be $C_{1}, \ldots, C_{k}$ and $l=\min \{j, k\}$. We shall regard $\sigma$ as a permutation of $[m+1, m+n]$. We shall construct $2 l+1$ noncrossing half-permutations $\pi \vee_{0} \sigma, \pi \vee_{1, o} \sigma, \pi \vee_{1, c} \sigma, \ldots, \pi \vee_{l, o} \sigma, \pi \vee_{l, c} \sigma$ in $N C L(m+n)$. The set $\left\{\pi \vee_{0} \sigma, \ldots, \pi \vee_{l, c} \sigma\right\}$ will be denoted $\pi * \sigma$, and called the convolution of $\pi$ and $\sigma$.

Let $\pi \vee_{0} \sigma$ be the permutation of $[m+n]$ obtained by letting $\pi$ act on $[m]$ and $\sigma$ act on $[m+1, m+n]$.

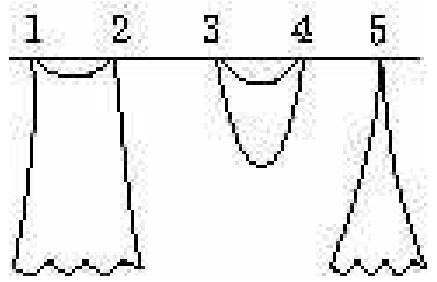

$\pi$

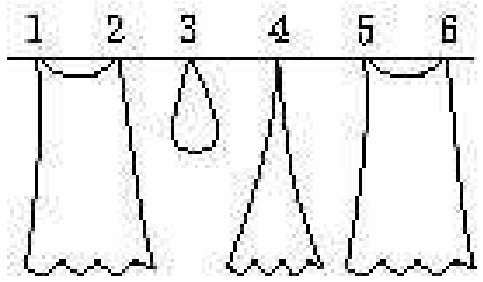

$\sigma$

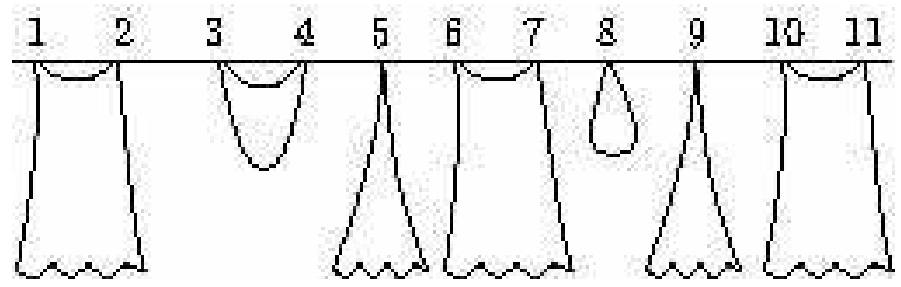

Figure 17. $\pi \vee_{0} \sigma$ is the concatenation of the two non crossing linear half-permutations.

In the figure above we have $\pi=\{(1,2),(3,4),(5)\}$ with $(1,2)$ and (5) open blocks; and $\sigma=\{(1,2),(3),(4),(5,6)\}$ with $(1,2),(4)$, and $(5,6)$ open blocks. We transfer $\sigma$ to the interval $[6,11]$ and obtain $\pi \vee_{0} \sigma=\{(1,2),(3,4),(5),(6,7),(8),(9),(10,11)\}$ with $(1,2),(5),(6,7)$, $(9)$, and $(10,11)$ open.

The open blocks of $\pi$ and $\sigma$ are ordered by their smallest element, which is the same as ordering by their largest element, as open blocks cannot be nested. The basic construction is to take the rightmost open block of $\pi, B_{j}=\left(p_{1}, \ldots, p_{r}\right)$ and the leftmost open block of $\sigma$, $C_{1}=\left(q_{1}, \ldots, q_{s}\right)$, and replace them with $\left(p_{1}, \ldots, p_{r}, q_{1}, \ldots, q_{s}\right)$ first as an open block then as a closed block. These permutations are called $\pi \vee_{1, o} \sigma$ and $\pi \vee_{1, c} \sigma$ respectively. We obtain $\pi \vee_{2, o} \sigma$ and $\pi \vee_{2, c} \sigma$ by applying this procedure (after $B_{j}$ and $C_{1}$ have been joined as a 
closed block) to the open blocks $B_{j-1}$ and $C_{2}$ and continuing until all the open blocks of one of the permutations are exhausted. This will produce $2 l+1$ permutations.

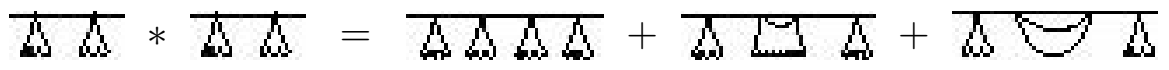 $+\sqrt{20}+$}

Lemma 46.

$$
\begin{aligned}
& W\left(d_{1} \otimes \cdots \otimes d_{m}\right)\left(e_{1} \otimes \cdots \otimes e_{n}\right) \\
& \quad-\psi\left(d_{m} e_{1}\right) W\left(d_{1} \otimes \cdots \otimes d_{m-1}\right)\left(e_{2} \otimes \cdots \otimes e_{n}\right) \\
& =d_{1} \otimes \cdots \otimes d_{m} \otimes e_{1} \otimes \cdots \otimes e_{n} \\
& +d_{1} \otimes \cdots \otimes d_{m} e_{1} \otimes \cdots \otimes e_{n}
\end{aligned}
$$

Proof. We prove the lemma by induction on $n$. By writing

$$
\begin{gathered}
e_{1} \otimes \cdots \otimes e_{n}=p\left(e_{1}\right) e_{2} \otimes \cdots \otimes e_{n}-e_{1} e_{2} \otimes \cdots \otimes e_{n} \\
-\psi\left(e_{1} e_{2}\right) e_{3} \otimes \cdots \otimes e_{n}-\psi\left(e_{1}\right) e_{2} \otimes \cdots \otimes e_{n}
\end{gathered}
$$

taking the adjoint of equation 19] and combining this with Corollary 44 we get that

$$
\begin{aligned}
& W\left(d_{1} \otimes \cdots \otimes d_{m}\right)\left(e_{1} \otimes \cdots \otimes e_{n}\right) \\
& -\psi\left(d_{m} e_{1}\right) W\left(d_{1} \otimes \cdots \otimes d_{m-1}\right)\left(e_{2} \otimes \cdots \otimes e_{n}\right) \\
& =W\left(d_{1} \otimes \cdots \otimes d_{m} \otimes e_{1}\right)\left(e_{2} \otimes \cdots \otimes e_{n}\right) \\
& -\psi\left(e_{1} e_{2}\right) W\left(d_{1} \otimes \cdots \otimes d_{m}\right)\left(e_{3} \otimes \cdots \otimes e_{n}\right) \\
& +W\left(d_{1} \otimes \cdots \otimes d_{m} e_{1}\right)\left(e_{2} \otimes \cdots \otimes e_{n}\right) \\
& -W\left(d_{1} \otimes \cdots \otimes d_{m}\right)\left(e_{1} e_{2} \otimes \cdots \otimes e_{n}\right)
\end{aligned}
$$

We may write

$$
\begin{aligned}
& W\left(d_{1} \otimes \cdots \otimes d_{m} e_{1}\right)\left(e_{2} \otimes \cdots \otimes e_{n}\right)-W\left(d_{1} \otimes \cdots \otimes d_{m}\right)\left(e_{1} e_{2} \otimes \cdots \otimes e_{n}\right) \\
&=\left\{W\left(d_{1} \otimes \cdots \otimes d_{m} e_{1}\right)\left(e_{2} \otimes \cdots \otimes e_{n}\right)\right. \\
&\left.-\psi\left(d_{m} e_{1} e_{2}\right) W\left(d_{1} \otimes \cdots \otimes d_{m-1}\right)\left(e_{3} \otimes \cdots \otimes e_{n}\right)\right\} \\
&-\left\{W\left(d_{1} \otimes \cdots \otimes d_{m}\right)\left(e_{1} e_{2} \otimes \cdots \otimes e_{n}\right)\right. \\
&\left.-\psi\left(d_{m} e_{1} e_{2}\right) W\left(d_{1} \otimes \cdots \otimes d_{m-1}\right)\left(e_{3} \otimes \cdots \otimes e_{n}\right)\right\}
\end{aligned}
$$

By applying our induction hypothesis to the bracketed terms we get $W\left(d_{1} \otimes \cdots \otimes d_{m}\right)\left(e_{1} \otimes \cdots \otimes e_{n}\right)$ 


$$
\begin{aligned}
& -\psi\left(d_{m} e_{1}\right) W\left(d_{1} \otimes \cdots \otimes d_{m-1}\right)\left(e_{2} \otimes \cdots \otimes e_{n}\right) \\
= & W\left(d_{1} \otimes \cdots \otimes d_{m} \otimes e_{1}\right)\left(e_{2} \otimes \cdots \otimes e_{n}\right) \\
& -\psi\left(e_{1} e_{2}\right) W\left(d_{1} \otimes \cdots \otimes d_{m}\right)\left(e_{3} \otimes \cdots \otimes e_{n}\right) \\
& +d_{1} \otimes \cdots \otimes d_{m} e_{1} \otimes \cdots \otimes e_{n}-d_{1} \otimes \cdots \otimes d_{m} \otimes e_{1} e_{2} \otimes \cdots \otimes e_{n}
\end{aligned}
$$

To conclude the proof we apply this formula $n-1$ times to obtain

$$
\begin{aligned}
W\left(d_{1} \otimes \cdots \otimes\right. & \left.d_{m}\right)\left(e_{1} \otimes \cdots \otimes e_{n}\right) \\
& \quad-\psi\left(d_{m} e_{1}\right) W\left(d_{1} \otimes \cdots \otimes d_{m-1}\right)\left(e_{2} \otimes \cdots \otimes e_{n}\right) \\
= & W\left(d_{1} \otimes \cdots \otimes e_{n}\right) \Omega+d_{1} \otimes \cdots \otimes d_{m} e_{1} \otimes \cdots \otimes e_{n}
\end{aligned}
$$

as required.

\section{Theorem 47 .}

$W_{\pi}\left(d_{1} \otimes \cdots \otimes d_{m}\right) W_{\sigma}\left(e_{1} \otimes \cdots \otimes e_{n}\right)=W_{\pi * \sigma}\left(d_{1} \otimes \cdots \otimes d_{m} \otimes e_{1} \otimes \cdots \otimes e_{n}\right)$

Proof. From the definition we only have to verify this in the special case that all the blocks of $\pi$ and $\sigma$ are open singletons. From the lemma above we have

$$
\begin{aligned}
& W\left(d_{1} \otimes \cdots \otimes d_{m}\right) W\left(e_{1} \otimes \cdots \otimes e_{n}\right) \\
& =d_{1} \otimes \cdots \otimes d_{m} \otimes e_{1} \otimes \cdots \otimes e_{n}+d_{1} \otimes \cdots \otimes d_{m} e_{1} \otimes \cdots \otimes e_{n} \\
& \quad+\psi\left(d_{m} e_{1}\right) W\left(d_{1} \otimes \cdots \otimes d_{m-1}\right) W\left(e_{2} \otimes \cdots \otimes e_{n}\right)
\end{aligned}
$$

Thus the theorem follows from repeated applications of lemma 46 above.

\section{THE CASE OF PAIRINGS}

In this paper we have only dealt with Wishart matrices, however there is a parallel program one can carry out for Gaussian matrices. Indeed Theorem 1 in the Gaussian case was obtained by CabanalDuvillard [CD] using stochastic integration. However a combinatorial proof along the lines presented here is equally possible. The idea is to consider only pairings throughout all the calculations. One has the notion of non-crossing circular and linear half-pairings; in this case the polynomials are the Chebyshev polynomials $\left\{C_{n}\right\}_{n}$ and $\left\{S_{n}\right\}_{n}$ of the first and second kind respectively renormalized to the interval $[-2,2]$. Exactly as in Corollary 44 one obtains the analogous result on Wick products needed in [MS], Lemma 5.5. Theorem 47llikewise has a similar formulation and proof. The pairing version of some of our results (e.g. Theorem 43) are special cases of Effros and Popa [EP], Thm 3.3. 


\section{ACKNOWLEDGMENTS}

The authors wish to thank Alexandru Nica for fruitful discussions at an early stage of this work.

\section{REFERENCES}

[A] M. Anshelevich, Appel Polynomials and their relatives, Inter. Math. Res. Notes, 2004, no. 65, 3469-3531.

[в] P. Biane, Some properties of crossings and partitions, Discrete Mathematics, 175, (1997), 41-53.

[CD] T. Cabanal-Duvillard, Fluctuations de la Loi Empirique de Grandes Matrices Aléatoires, Ann. I. H. Poincaré (B), Probabilités et Statistiques, 37 (2001) $373-402$.

[EP] E. G. Effros and M. Popa, Feynman diagrams and Wick Products associated with $q$-Fock space, Proc. Nat. Acad. Sci. (USA), 100, 8629 - 8633.

[Јон] K. Johansson, On Fluctuations of Eigenvalues of Random Hermitian Matrices, Duke Math. J., 91 ( 1998), 151 - 204.

[JON] D. Jonsson Some limit theorems for the eigenvalues of a sample covariance matrix, J. Mult. Anal., 12 (1982), 1-38.

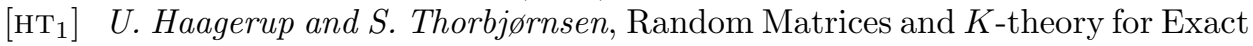
C*-algebras, Documenta Mathematica, 4 (1999), 341 - 450.

$\left[\mathrm{HT}_{2}\right]$ Uffe Haagerup and S. Thorbjørnsen, Random Matrices with Complex Gaussian Entries, Expositiones Math., 21 (2003), 293 - 337.

[HP] F. Hiai and D. Petz, The Semicircle Law, Free Random Variables and Entropy, Providence, R.I., Amer. Math. Soc., 2000.

[J] $\quad V . F . R$. Jones, The annular structure of subfactors, L'Enseignement Mathematique, to appear, math.OA/0105071

[MN] J. Mingo and A. Nica, Annular non-crossing permutations and partitions, and second-order asymptotics for random matrices, Inter. Math. Res. Notices, 2004, $\mathrm{n}^{\circ} 28,1413-1460$.

[MS] J. Mingo and R. Speicher, Second order freeness and fluctuations of random matrices: I. Gaussian and Wishart matrices and cyclic Fock spaces, Preprint, 2004, math/OA/0405191.

[Mśs] J. Mingo, P. Śniady, and R. Speicher Second Order Freeness and Fluctuations of Random Matrices: II. Unitary Random Matrices, preprint May 2004, 22 pages. math/OA/0405258

Queen's University, Department of Mathematics and Statistics, JefFERY Hall, Kingston, ON K7L 3N6, Canada

E-mail address: 2tpk@qlink.queensu.ca

E-mail address: mingo@mast.queensu.ca

E-mail address: speicher@mast .queensu.ca 